\title{
A DISK OF YOUNG STARS AT THE GALACTIC CENTER AS DETERMINED BY INDIVIDUAL STELLAR ORBITS
}

\author{
J. R. Lu ${ }^{1}$, A. M. Ghez ${ }^{1,2}$, S. D. Hornstein ${ }^{1,3}$, M. R. Morris ${ }^{1}$, E. E. Becklin ${ }^{1}$, And K. Matthews ${ }^{4}$ \\ ${ }^{1}$ UCLA Department of Physics and Astronomy, Los Angeles, CA 90095-1562, USA; jlu@astro.ucla.edu, morris@astro.ucla.edu, and becklin@astro.ucla.edu \\ ${ }^{2}$ UCLA Institute of Geophysics and Planetary Physics, Los Angeles, CA 90095-1565, USA; ghez@astro.ucla.edu \\ ${ }^{3}$ Center for Astrophysics \& Space Astronomy, Department of Astrophysical and Planetary Sciences, University of Colorado, Boulder, CO 80309, USA; \\ seth.hornstein@colorado.edu \\ ${ }^{4}$ Caltech Optical Observatories, California Institute of Technology, MS 320-47, Pasadena, CA 91125, USA; kym@caltech.edu \\ Received 2008 June 9; accepted 2008 August 27; published 2008 December 22
}

\begin{abstract}
We present new proper motions from the $10 \mathrm{~m}$ Keck telescopes for a puzzling population of massive, young stars located within 3 ".5 $(0.14 \mathrm{pc})$ of the supermassive black hole at the Galactic center. Our proper motion measurements have uncertainties of only 0.07 mas $\mathrm{yr}^{-1}\left(3 \mathrm{~km} \mathrm{~s}^{-1}\right)$, which is $\gtrsim 7$ times better than previous proper motion

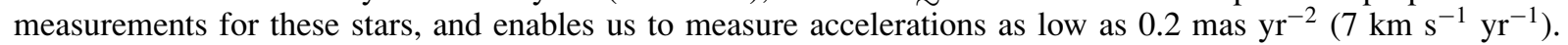
Using these measurements, line-of-sight velocities from the literature, and three-dimensional velocities for additional young stars in the central parsec, we constrain the true orbit of each individual star and directly test the hypothesis that the massive stars reside in two stellar disks as has been previously proposed. Analysis of the stellar orbits reveals only one of the previously proposed disks of young stars using a method that is capable of detecting disks containing at least seven stars. The detected disk contains $50 \%$ of the young stars, is inclined by $\sim 115^{\circ}$ from the plane of the sky, and is oriented at a position angle of $\sim 100^{\circ}$ east of north. Additionally, the on-disk and off-disk populations have similar $K$-band luminosity functions and radial distributions that decrease at larger radii as $\propto r^{-2}$. The disk has an out-of-the-disk velocity dispersion of $28 \pm 6 \mathrm{~km} \mathrm{~s}^{-1}$, which corresponds to a half-opening angle of $7^{\circ} \pm 2^{\circ}$, and several candidate disk members have eccentricities greater than 0.2 . Our findings suggest that the young stars may have formed in situ but in a more complex geometry than a simple, thin circular disk.
\end{abstract}

Key words: black hole physics - Galaxy: center - infrared: stars - techniques: high angular resolution

Online-only material: color figures, extended figure set

\section{INTRODUCTION}

The center of our Galaxy harbors not only a supermassive black hole (Sgr A*, $M_{\bullet} \sim 4 \times 10^{6} M_{\odot}$; Eckart \& Genzel 1996; Genzel et al. 1996; Ghez et al. 1998, 2000, 2003, 2005a; Schödel et al. 2002, 2003; Eisenhauer et al. 2005), but also a population of massive (10-120 $\left.M_{\odot}\right)$, young $(\lesssim 10-100 \mathrm{Myr})$ stars whose existence is a puzzle. The origin of such young stars has been difficult to explain since the gas densities observed today are orders of magnitude too low for a gas clump to overcome the extreme tidal forces and collapse to form stars (e.g., Sanders 1992; Morris 1993; Ghez et al. 2005a; Alexander 2005, for reviews). And yet, within the central parsec of our Galaxy, nearly 100 stars have been classified as OB main-sequence stars, more luminous $\mathrm{OB}$ giants and supergiants, and post-main-sequence Wolf-Rayet stars (Allen et al. 1990; Krabbe et al. 1991; Blum et al. 1995; Krabbe et al. 1995; Tamblyn et al. 1996; Najarro et al. 1997; Ghez et al. 2003; Paumard et al. 2006), with the more evolved massive stars having ages as young as $6 \pm 2 \mathrm{Myr}$ (Paumard et al. 2006). Populations of young stars have also been observed in the nuclei of other galaxies, such as M31 (Bender et al. 2005), suggesting that star formation near a supermassive black hole may be a common, but not understood, phenomenon in galaxy evolution. The close proximity of the black hole at the center of the Milky Way provides a unique laboratory for studying this "paradox of youth" (e.g., Ghez et al. 2003, 2005a; Schödel et al. 2003; Eisenhauer et al. 2005).

Proposed resolutions to the paradox of youth can be grouped into several broad categories, including (1) rejuvenation of an older population such that older stars appear young,
(2) dynamical migration from larger radii, and (3) in situ formation. Rejuvenation scenarios include stripping (Davies et al. 1998; Davies \& King 2005), or tidal heating of the atmospheres of old stars (Alexander \& Morris 2003), or combining multiple low mass stars via collisional mergers to form a higher mass hot star akin to a "blue straggler" (Lee 1996; Morris 1993; Genzel et al. 2003). Although these processes may be candidates for explaining the closest young stars within the central arcsecond, they cannot account for the OB giants, OB supergiants, and Wolf-Rayet stars that are located at larger radii $\left(1^{\prime \prime}-14^{\prime \prime}\right)$, since the rate of collisions is too low to produce the observed total numbers. Thus, it appears that these massive young stars must have formed, or were deposited, in the central region within the last 4-8 Myr. Dynamical migration scenarios attempt to resolve the paradox of youth with the formation of a massive star cluster at larger distances from the black hole (3-30 pc). Such a cluster would spiral in due to dynamical friction and deposit stars at smaller radii where they are observed today (Gerhard 2001). However, for a cluster to reach the central parsec in only a few million years, it must be very massive and centrally concentrated (Kim \& Morris 2003; Portegies Zwart et al. 2003; McMillan \& Portegies Zwart 2003; Gürkan \& Rasio 2005), and it may even require the existence of an intermediate-mass black hole (IMBH) as an anchor in the cluster core (Hansen \& Milosavljević 2003; Kim et al. 2004). In situ, star-formation scenarios can resolve the paradox of youth if a massive, selfgravitating gas disk was once present around the black hole (Levin \& Beloborodov 2003). Such a disk would be sufficiently dense to overcome the strong tidal forces, and gravitational instabilities would then lead to fragmentation and the formation of 
stars, as has been suggested in the context of both the Galactic center circumnuclear disk and active galactic nucleus (AGN) accretion disks in other galaxies (e.g., Kolykhalov \& Syunyaev 1980; Shlosman \& Begelman 1989; Morris \& Serabyn 1996; Sanders 1998; Goodman 2003; Nayakshin \& Cuadra 2005).

Insight into the origins of the massive, young stars may be obtained through observations of the spatial distribution and stellar dynamics of this population. Already, high-resolution infrared imaging and spectroscopy have shown that the young stars between 0 .' 5 and $14^{\prime \prime}(0.02-0.6 \mathrm{pc})$ exhibit coherent rotation (Genzel et al. 2000). Analyses of the statistical properties of the three-dimensional velocity vectors for these stars suggest that they may reside in two disks. The first proposed disk has a clockwise sense of rotation, as projected onto the plane of the sky (Levin \& Beloborodov 2003, hereafter clockwise-rotating or $\mathrm{CW}$ disk), while the second proposed disk is counterclockwiserotating (CCW; Genzel et al. 2003) and is nearly perpendicular to the first. The proposed disks extend from $\sim 0^{\prime \prime} .8$ to at least $7^{\prime \prime}$ (Paumard et al. 2006). Other velocity vector analyses show that there are possible comoving groups or clusters of stars, including the IRS 13 cluster, which is proposed to lie within the putative CCW disk (Maillard et al. 2004; Schödel et al. 2005), and the IRS 16SW comoving group, which are also consistent with the proposed CW disk (Lu et al. 2005). The two proposed disks are inferred to be oriented with an inclination and angle to the ascending node of $\left(i_{\mathrm{CW}}=127^{\circ} \pm 2^{\circ}, \Omega_{\mathrm{CW}}=99^{\circ} \pm 2^{\circ}\right)$ and $\left(i_{\mathrm{CCW}}=24^{\circ} \pm 4^{\circ}, \Omega_{\mathrm{CCW}}=167^{\circ} \pm 7^{\circ}\right)$ and to have a finite angular thickness of $\Delta \theta_{\mathrm{CW}} \sim 14^{\circ}$ and $\Delta \theta_{\mathrm{CW}} \sim 19^{\circ}$, where $\Delta \theta$ is the standard deviation of the orbital inclinations distributed normally about the disk plane (Paumard et al. 2006). The thickness of the stellar disks has been attributed to thickening as a result of gravitational interactions between the two disks, which provides an estimate of the disk masses (Nayakshin et al. 2006). The derived mass is smaller than the mass inferred from the number of observed young stars, assuming a Salpeter initial mass function (IMF); accordingly, Nayakshin et al. (2006) suggest that the disks have a top-heavy mass function. Both in situ gas disk and inspiraling star cluster formation scenarios have been used to explain the kinematics of this young star population and to predict that the stars should lie in a common orbital plane. However, the presence of two stellar disks with similarly aged populations requires either two nearly concurrent gas disks or two infalling star clusters; and both of these scenarios are difficult to produce. Therefore, to understand the recent starformation history, it is critical to measure the orbital planes of individual stars in order to confirm the existence of the two stellar disks previously derived from a statistical analysis of velocity vectors alone.

The in situ gas disk and inspiraling star cluster formation scenarios predict different structures and evolutions for the resulting stellar disk, particularly with respect to the eccentricities and radial distribution of stars within the disk. Early models of a self-gravitating gas disk around the supermassive black hole at the center of the Milky Way produce stars with a steep radial profile in the disk surface density, $\Sigma \propto r^{\alpha}$, with $\alpha \sim-2$ (Lin $\&$ Pringle 1987; Levin 2007). These models typically result in stars on circular orbits as would be the case for the slow build up of a gas disk that is circularized before there is sufficient mass for gravitational instabilities to set in (Milosavljević \& Loeb 2004; Nayakshin \& Cuadra 2005; Levin 2007). The stellar eccentricities of an initially circular disk can relax to higher eccentricities up to $e_{\mathrm{rms}}=\sqrt{\left\langle e^{2}\right\rangle} \sim 0.15$ for a normal IMF or $e_{\text {rms }} \sim 0.3$ for a top-heavy IMF (Alexander et al. 2007; Cuadra et al. 2008). More recent models have also shown that star formation can occur rapidly before circularization in an initially eccentric disk as might result from the infall of a single massive molecular cloud or a cloud-cloud collision (Sanders 1998; Nayakshin et al. 2007; Alexander et al. 2008). These eccentric self-gravitating accretion disk models typically produce a more top-heavy IMF than initially circular disks. Alternatively, an inspiraling star cluster would dissolve into a disk of stars with a flatter radial profile $\left(\Sigma \propto r^{-0.75}\right.$; Berukoff \& Hansen 2006) whose orbital eccentricities would reflect the eccentricity of the cluster's orbit, which could be either circular or eccentric (Portegies Zwart et al. 2003; McMillan \& Portegies Zwart 2003; Kim \& Morris 2003; Kim et al. 2004; Gürkan \& Rasio 2005; Berukoff \& Hansen 2006). Previous measurements of the radial distribution of young stars yield a steep radial profile consistent with in situ formation (Paumard et al. 2006). Also, the eccentricities of the stars have previously been estimated from observations by assuming that the stars orbit in a disk; however, there are conflicting results claiming that the stars in the clockwise-rotating disk are on nearly circular orbits (Paumard et al. 2006) or on eccentric orbits (Beloborodov et al. 2006). Determining the radial profile and stellar eccentricities of stars in a disk may provide observational constraints on the origin of the young stars.

We present an improved proper motion study that yields an order of magnitude more precise proper motions and the first measurement of accelerations in the plane of the sky for stars outside the central arcsecond. By combining the stellar positions, proper motions, radial velocities, and accelerations, we estimate stellar orbital parameters and test whether the young stars reside on one or two stellar disks in a more direct manner than previous methods using only velocity information. This provides a direct test of the existence, membership, and properties of these disks. The observations are described in Section 2 and the astrometric analysis procedure and results are detailed in Section 3. Orbit analysis and results are presented in Sections 4 and 5 and a discussion of the implications for the origin of the massive, young stars at the Galactic center is presented in Section 6.

\section{OBSERVATIONS}

This study utilizes 29 epochs of high-resolution, infrared images of the Galaxy's central stellar cluster, which were taken from 1995 to 2005 using both speckle and laser guide star adaptive optics (LGS AO) observing techniques on the W. M. Keck $10 \mathrm{~m}$ telescopes. These data sets are listed in Table 1 and all but the additional LGS AO observation from 2005 are described in detail in earlier papers (Ghez et al. 1998, 2000, 2005a; Lu et al. 2005; Rafelski et al. 2007). Columns 3 and 4 list the individual exposure times and the total number of frames for each epoch of data. All 27 speckle imaging observations were taken using the facility near-infrared camera (NIRC; Matthews \& Soifer 1994; Matthews et al. 1996), which has a plate scale of $\sim 20$ mas pixel $^{-1}$, and a $5^{\prime \prime} .22 \times 5^{\prime \prime}$.22 field of view. The two adaptive optics imaging observations used the facility LGS AO system (Wizinowich et al. 2006; van Dam et al. 2006) and the near-infrared camera, NIRC2 (PI: K. Matthews) with a plate scale of $9.963 \pm 0.006$ mas pixel $^{-1}$ (Ghez et al. 2008) and a $10^{\prime \prime} .2 \times 10^{\prime \prime} .2$ field of view. While the laser guide star is used to correct most of the atmospheric aberrations, the low-order, tiptilt terms were corrected using visible observations of USNO 0600-28577051 $\left(R=13.7 \mathrm{mag}\right.$ and $\left.\Delta r_{\mathrm{Sgr} \mathrm{A} *}=19^{\prime \prime}\right)$. 
Table 1

List of Observations

\begin{tabular}{|c|c|c|c|c|c|c|c|c|c|}
\hline Date $^{\mathrm{a}}$ & Filter $^{b}$ & $\begin{array}{l}t_{\mathrm{exp}, i} \\
(\mathrm{~s})\end{array}$ & $\begin{array}{c}\text { Frames }^{\mathrm{c}} \\
\text { Used }\end{array}$ & $\begin{array}{c}\text { FWHM } \\
\text { (mas) }\end{array}$ & Strehl & $\begin{array}{l}\text { Number } \\
\text { of Stars }\end{array}$ & $\begin{array}{c}K_{\text {turnover }}^{\mathrm{d}} \\
\text { (mag) }\end{array}$ & $\begin{array}{l}\text { Pos. Error }{ }^{\mathrm{e}} \\
\text { (mas) }\end{array}$ & Data Source ${ }^{f}$ \\
\hline 1995.439 & $K$ & 0.12 & 1562 & 58 & 0.06 & 124 & 15.2 & 1.1 & speckle; (ref. 1) \\
\hline 1996.485 & $K$ & 0.13 & 857 & 60 & 0.03 & 71 & 13.5 & 1.7 & speckle; (ref. 1) \\
\hline 1997.367 & $K$ & 0.13 & 1834 & 61 & 0.05 & 116 & 15.2 & 1.1 & speckle; (ref. 1) \\
\hline 1998.251 & $K$ & 0.15 & 1645 & 62 & 0.04 & 81 & 12.9 & 1.4 & speckle; (ref. 2) \\
\hline 1998.366 & $K$ & 0.14 & 2096 & 69 & 0.05 & 120 & 15.1 & 1.2 & speckle; (ref. 2) \\
\hline 1998.505 & $K$ & 0.14 & 936 & 63 & 0.07 & 101 & 15.6 & 1.7 & speckle; (ref. 2) \\
\hline 1998.590 & $K$ & 0.14 & 1914 & 62 & 0.06 & 139 & 15.5 & 0.9 & speckle; (ref. 2) \\
\hline 1998.771 & $K$ & 0.14 & 1085 & 56 & 0.07 & 111 & 15.4 & 1.1 & speckle; (ref. 2) \\
\hline 1999.333 & $K$ & 0.14 & 1848 & 72 & 0.08 & 136 & 15.5 & 1.1 & speckle; (ref. 2) \\
\hline 1999.559 & $K$ & 0.14 & 2092 & 57 & 0.10 & 141 & 15.6 & 0.8 & speckle; (ref. 2) \\
\hline 2000.305 & $K$ & 0.14 & 1471 & 56 & 0.03 & 62 & 13.5 & 1.6 & speckle; (ref. 3) \\
\hline 2000.381 & $K$ & 0.14 & 2180 & 56 & 0.09 & 142 & 15.6 & 0.9 & speckle; (ref. 3) \\
\hline 2000.548 & $K$ & 0.14 & 1572 & 63 & 0.07 & 132 & 15.6 & 1.2 & speckle; (ref. 3) \\
\hline 2000.797 & $K$ & 0.14 & 1506 & 60 & 0.04 & 77 & 14.0 & 1.8 & speckle; (ref. 3) \\
\hline 2001.351 & $K$ & 0.14 & 1979 & 56 & 0.07 & 137 & 15.5 & 0.9 & speckle; (ref. 3) \\
\hline 2001.572 & $K$ & 0.14 & 1687 & 57 & 0.12 & 141 & 15.6 & 1.1 & speckle; (ref. 3) \\
\hline 2002.309 & $K$ & 0.14 & 1957 & 67 & 0.06 & 137 & 15.5 & 1.0 & speckle; (ref. 3) \\
\hline 2002.391 & $K$ & 0.14 & 1433 & 60 & 0.09 & 141 & 15.5 & 0.8 & speckle; (ref. 3) \\
\hline 2002.547 & $K$ & 0.14 & 1137 & 63 & 0.06 & 115 & 14.3 & 1.7 & speckle; (ref. 3) \\
\hline 2003.303 & $K$ & 0.14 & 1815 & 62 & 0.04 & 119 & 15.2 & 1.2 & speckle; (ref. 3) \\
\hline 2003.554 & $K$ & 0.14 & 1713 & 65 & 0.07 & 134 & 15.7 & 1.5 & speckle; (ref. 3) \\
\hline 2003.682 & $K$ & 0.14 & 1780 & 65 & 0.07 & 130 & 15.3 & 1.1 & speckle; (ref. 3) \\
\hline 2004.327 & $K$ & 0.14 & 1444 & 63 & 0.09 & 136 & 15.6 & 1.0 & speckle; (ref. 4) \\
\hline 2004.564 & $K$ & 0.14 & 2156 & 60 & 0.07 & 143 & 15.5 & 1.1 & speckle; (ref. 4) \\
\hline 2004.567 & $K^{\prime}$ & 9 & 12 & 60 & 0.31 & 145 & 15.8 & 1.0 & LGSAO; (ref. 5) \\
\hline 2004.660 & $K$ & 0.14 & 1300 & 59 & 0.08 & 114 & 15.2 & 1.3 & speckle; (ref. 4) \\
\hline 2005.312 & $K$ & 0.14 & 1677 & 60 & 0.07 & 132 & 15.3 & 1.0 & speckle; (ref. 6) \\
\hline $2005.495^{\mathrm{g}}$ & $K_{\mathrm{CO}}, K_{\mathrm{cont}}$ & $36,59.5$ & 10 & 61 & 0.32 & 146 & 15.7 & 1.2 & LGSAO; (new) \\
\hline 2005.566 & $K$ & 0.14 & 1825 & 62 & 0.05 & 113 & 15.1 & 1.7 & speckle; (ref. 6) \\
\hline
\end{tabular}

Notes.

a Dates are computed as the weighted average of UT dates from the individual exposures.

${ }^{\mathrm{b}}$ Filters used include $K\left(\lambda_{o}=2.2 \mu \mathrm{m}, \Delta \lambda=0.4 \mu \mathrm{m}\right), K^{\prime}\left(\lambda_{o}=2.12 \mu \mathrm{m}, \Delta \lambda=0.35 \mu \mathrm{m}\right), K_{\mathrm{CO}}\left(\lambda_{o}=2.289 \mu \mathrm{m}\right.$, $\Delta \lambda=0.048 \mu \mathrm{m})$, and $K_{\text {cont }}\left(\lambda_{o}=2.270 \mu \mathrm{m}, \Delta \lambda=0.030 \mu \mathrm{m}\right)$.

$\mathrm{c}$ The number of frames used in the final combined image.

d The turnover of the number of stars at a given magnitude provides a rough estimate of the completeness limit.

e The average positional uncertainty due to centroiding in each epoch is estimated from a set of 25 stars detected in all epochs and brighter than $K<13$. The two LGSAO epoch positional errors include an additional term of 0.88 mas to account for residual distortion.

${ }^{f}$ Data originally reported in (1) Ghez et al. (1998), (2) Ghez et al. (2000), (3) Ghez et al. (2005a), (4) Lu et al. (2005), (5) Ghez et al. (2005b), and (6) Rafelski et al. (2007).

g Five exposures were taken in each of two narrowband filters with different exposure times, but similar sensitivity and astrometric precision. All frames from both filters were combined in order to extract astrometric measurements from this data set.

In addition to the 27 speckle observation and the 2004 LGS AO observations described in previous works, a new LGS AO data set was obtained in 2005 June. This data set was taken using two different narrowband filters, $K_{\mathrm{CO}}\left(\lambda_{o}=2.289 \mu \mathrm{m}, \Delta \lambda=\right.$ $0.027 \mu \mathrm{m})$ and $K_{\text {cont }}\left(\lambda_{o}=2.270 \mu \mathrm{m}, \Delta \lambda=0.030 \mu \mathrm{m}\right)$, rather than the $K^{\prime}$ broadband filter used for the 2004 LGS AO observations. For each filter, images were taken in a five-position pattern around a 4". 0 box with exposure times of $36 \mathrm{~s}\left(t_{\exp }=7.2 \mathrm{~s}\right.$, five co-adds) and $59.5 \mathrm{~s}\left(t_{\text {exp }}=11.9 \mathrm{~s}\right.$, five co-adds $)$ for the $K_{\mathrm{CO}}$ and $K_{\text {cont }}$ filters, respectively. The choice of narrowband filters was driven by a different project and the data sets from the two filters were combined together for the present study (see Section 3.1). Resulting Strehl ratios were $\sim 0.25-0.35$ in the individual frames.

\section{ASTROMETRIC DATA ANALYSIS AND RESULTS}

The goal of this analysis is to obtain high-precision astrometry for a sample of young stars that are candidate disk members and have existing radial velocity measurements. Based on spectroscopic identification, there are currently 90 known young stars with radial velocity measurements listed in Paumard et al. (2006) based on high-quality ("quality 1 or 2") spectral classifications. We define a primary sample that includes those known young stars found in our astrometric data sets that have projected radii between 0 .' 8 and 3 .'5. The inner radius is set by the proposed inner edge of the clockwise disk of young stars and young stars interior to this radius are on more randomly oriented orbits (Ghez et al. 2005a; Eisenhauer et al. 2005). The outer radius is set by the field of view of the speckle data sets. Over this region, Paumard et al. (2006) note that all young stars brighter than $K=13.5$ should be identified, which includes OB giants and supergiants. A total of 32 such young stars are in our 11 years astrometric data set and comprise the sample for this study. Of the 32 stars in our sample, 23 are among the 36 stars thought to be part of the clockwise disk, 2 are among the 12 candidate members of the counterclockwise disk, and the 
remaining 7 are among the 42 stars not assigned to either disk by Paumard et al. (2006).

We also define an extended sample that includes both the primary sample of 32 stars and an additional 41 young stars found by Paumard et al. (2006) at larger radii that are outside the field of view of our astrometric measurements. The astrometry for the additional 41 stars is taken from Paumard et al. (2006), which has an order of magnitude lower precision and lacks any constraints on the accelerations. However, we use the extended sample to explore the kinematics of the young stars at larger radii with the same analysis techniques used on the primary sample. We also note that the spectroscopic observations used to identify the young stars at larger radii were taken in a different setup than in the central regions, with lower spectral resolution and lower Strehl; thus the completeness limit may be somewhat brighter in this region. However, any difference is statistically insignificant given that a two-sample $\mathrm{KS}$ test yields a $50 \%$ probability that the primary sample and those additional stars added to the extended sample have the same $K$-band luminosity function (KLF). The extended sample is used only to supplement our analysis; therefore, to avoid confusion, all analysis and results are reported for the primary sample, which has more precise proper motions and accelerations, unless specifically noted otherwise.

Astrometric positions for the young stars in the primary sample are extracted from the imaging data sets listed in Table 1 using similar techniques to those described in Ghez et al. (1998, 2000), Lu et al. (2005), and Ghez et al. (2005a), with the following key changes: (1) geometric distortion is corrected in the speckle images using an improved distortion solution (see Section 3.1, Appendix A), (2) speckle images are combined with an improved algorithm developed and implemented by Hornstein (2007), and (3) image coordinates are transformed between data sets with more degrees of freedom (see Section 3.2). Sections 3.1 and 3.2 describe the analysis in detail and Section 3.3 presents the astrometric results.

\subsection{Image Processing}

To achieve precise astrometry, the basic image reduction steps, particularly geometric distortion correction, must be carefully implemented. First, both speckle and LGS AO individual exposures are processed using standard techniques of sky subtraction, flat-fielding, and bad pixel correction. Next, the images are transformed to correct for optical distortion. For the LGS $\mathrm{AO} / \mathrm{NIRC} 2$ images, optical distortions are well characterized at the $\sim 2$ milliarcsec level over $2^{\prime \prime}$ (Ghez et al. 2008, Appendix A) by the pre-ship review distortion coefficients ${ }^{5}$ and the distortions are removed from the images using the IRAF routine, Drizzle (Fruchter \& Hook 2002). The speckle images, obtained with NIRC, have a known off-axis distortion that can be corrected as described in Ghez et al. (1998). However, this distortion solution does not account for any distortion introduced by the additional optics in the NIRC reimager, which magnifies the image scale by a factor of $\sim 7$ from seeing limited sampling to diffraction-limited sampling. Speckle data sets were acquired in such a way as to minimize the effects of this residual distortion in the center of the field of view and have resulting residual distortion errors that are smaller than the typical centroiding error, which is $\sim 2$ mas, for stars at radii $<0$ '.5. However, astrometric uncertainties for stars outside this region are dominated by the uncorrected distortion, which grows to $\sim 6$ mas near the field

\footnotetext{
5 http://www2.keck.hawaii.edu/inst/nirc2/
}

edge at a radius of 2.5 (Ghez et al. 2005a). In order to characterize the residual distortion in NIRC, simultaneous images of the Galactic center were obtained with both NIRC and NIRC2 with the NIRC2 images serving as a reference coordinate system (see Appendix A). The speckle image distortion is mapped by comparing stars' positions in both NIRC and NIRC2 images. As shown in Appendix A, the resulting NIRC to NIRC2 transformation is characterized at the $\sim 2$ mas level over the entire field of view.

After distortion correction, individual exposures are combined into a final diffraction-limited image using different methods for speckle and LGS AO data sets. Speckle images are produced by first rejecting the low Strehl ratio frames (typically $75 \%$ of frames are rejected) and then stacking the remaining frames using a weighted shift-and-add (SAA) routine (Hornstein 2007). The resulting combined images have a point-spread function (PSF) composed of a diffraction-limited core (FWHM $\sim 0^{\prime \prime} 055$ ) on top of a broad seeing halo (FWHM $\sim 0$.'4). The improved image combination algorithm attempts to maximize the signal-to-noise ratio $(\mathrm{S} / \mathrm{N})$ of the final image while preserving the highest spatial resolution. Quantitatively, the weighted SAA method doubles the fraction of light contained in the diffraction-limited core (from $3.5 \%$ to $7.0 \%$ ) over the standard SAA scheme with no weighting and no frame rejection (Hornstein 2007). The LGS AO individual exposures are all of similar quality and are thus all averaged together, without weighting, in order to produce the final high-resolution image for each data set. Although the 2005 June data were taken in two different filters $\left(K_{\mathrm{CO}}\right.$ and $\left.K_{\text {cont }}\right)$, all the images were combined together to increase the final $\mathrm{S} / \mathrm{N}$. While photometry from this epoch is marginally impacted, the astrometry is comparable to other epochs. Each data set was also subdivided to produce three equivalent quality (randomized in time) subsets to make three images used for determining photometric and astrometric uncertainties. The resulting images are summarized in Table 1, including the achieved spatial resolution (FWHM) and the Strehl ratio.

\subsection{Stellar Positions and Coordinate Transformations}

In order to extract astrometric information for the sample of young stars, the coordinate system from each data set is transformed into a common reference frame using the stars in each image to determine the transformation parameters. Since the accuracy of this transformation relies on the assumption that there is no net rotation of the sample, we use all stars detected in each data set, not just the young stars, in this analysis. The steps for (1) measuring stars' positions in each epoch, (2) transforming to a common (relative) reference frame, and (3) determining the absolute coordinate system are described below and utilize all stars detected in the data sets; then as a final step, the young star sample is extracted.

In each data set, stars are identified and their positions measured using the IDL PSF fitting routine "StarFinder" (Diolaiti et al. 2000). StarFinder generates a PSF from several bright stars in the field and cross-correlates the resulting PSF with the image. The PSF was iteratively constructed using IRS 16C, $16 \mathrm{NW}$, and S2-17 for the speckle maps and IRS $16 \mathrm{C}, 16 \mathrm{NW}$, $16 \mathrm{NE}, 16 \mathrm{SW}, 33 \mathrm{E}, 33 \mathrm{~W}, 7,29 \mathrm{~N}$, and GEN+2.33+4.60 for the LGS AO images. Candidate stars are those for which StarFinder correlation peaks have a correlation value higher than 0.8 and positions and fluxes are extracted by fitting the PSF to each correlation peak. From the candidate star list, spurious detections are then eliminated by requiring that each star be detected in all 
three of the subset images with a correlation of higher than 0.6. The positional centroiding uncertainties for each candidate star are estimated from the root mean square (rms) of their locations in the three subset images, and an additional systematic error term of 0.88 mas is added in quadrature to all stars in LGS $\mathrm{AO}$ epochs to account for residual distortion in the central $5^{\prime \prime}$ of NIRC2 (Ghez et al. 2008). The candidate stars are flux calibrated using the apparent magnitudes of the nonvariable stars, IRS 16C, IRS 16SW-E, S2-17, S1-23, S1-3, S1-4, S2-22, S2-5, S1-68, S0-13, and S1-25, as measured by Rafelski et al. (2007). The star detections from each epoch are cross-identified with stars from all other epochs and those stars that are detected in at least 16 out of 29 epochs are used to create a master star list. The threshold of 16 or more epochs is used in order to insure high astrometric precision; for a threshold of less than 16 epochs, the number of detected stars rises dramatically as does the number of sources showing significant $(\gtrsim 3 \sigma)$ accelerations in nonphysical directions, indicating a high frequency of false detections (see Section 3.3 for further discussion). Stars in the master list are also examined for source confusion, which may occur when two stars pass close enough to each other such that StarFinder only detects a single source with biased astrometry rather than detecting both stars. Source measurements from individual epochs are rejected if two stars pass within 55 mas ( $\sim 1$ spatial resolution element) of each other and only one source is detected by StarFinder. The results of this stage of the analysis are summarized in Table 1, which provides for each data set the average centroiding error for the brightest stars $(K<13$; also see Figure 1) and the sensitivity as estimated by the peak in a histogram of the $K$-band magnitudes (bins $=0.1 \mathrm{mag}$ ) of all the stars in the data set. Averaged over all stars in all maps, the centroiding uncertainties have a mean value of 1.6 mas for the brightest stars ( $K \leqslant 13 \mathrm{mag}$ ) and 3.4 mas for the fainter stars $(13<K<16 \mathrm{mag})$.

The coordinate system for each image is transformed to a common local reference frame defined by the 2004 July LGS AO/NIRC2 image's coordinates and pixel scale. This particular LGS AO epoch was chosen as the reference because the NIRC speckle distortion solution is tied to this epoch, thus providing a smooth transition between speckle and LGS AO data sets. The procedure for deriving the coordinate transformation for all of the data sets is nontrivial, since the stars in the images have detectable motions. Optimal alignment is achieved by minimizing the error-weighted, net displacement for all the stars as described by Ghez et al. (1998) while allowing for translation, rotation, and two magnifications in arbitrary, but perpendicular, directions. This is a higher-order transformation than was used in our earlier astrometric works, which only allowed for translation and rotation. The new transformation equations have the form

$$
\begin{gathered}
x_{\text {pix }}=a_{0}+a_{1} x_{\text {pix }}^{\prime}+a_{2} y_{\text {pix }}^{\prime} \\
y_{\text {pix }}=b_{0}+b_{1} y_{\text {pix }}^{\prime}+b_{2} x_{\text {pix }}^{\prime},
\end{gathered}
$$

where $x_{\text {pix }}^{\prime}$ and $y_{\text {pix }}^{\prime}$ are the input detector coordinates in pixels and $x_{\text {pix }}$ and $y_{\text {pix }}$ are the output coordinates for each star, and all other variables are free parameters that are common across all stars in the alignment fit. As in Ghez et al. (2005a), stars within 0.5 of Sgr A* are excluded from the transformation as they exhibit large nonlinear motions. Additionally, all spectroscopically identified young stars are excluded from the transformation as they have a known net rotation (Genzel et al. 2000). Initially, each image is aligned to the reference image by assuming that the stars have no proper motions and finding
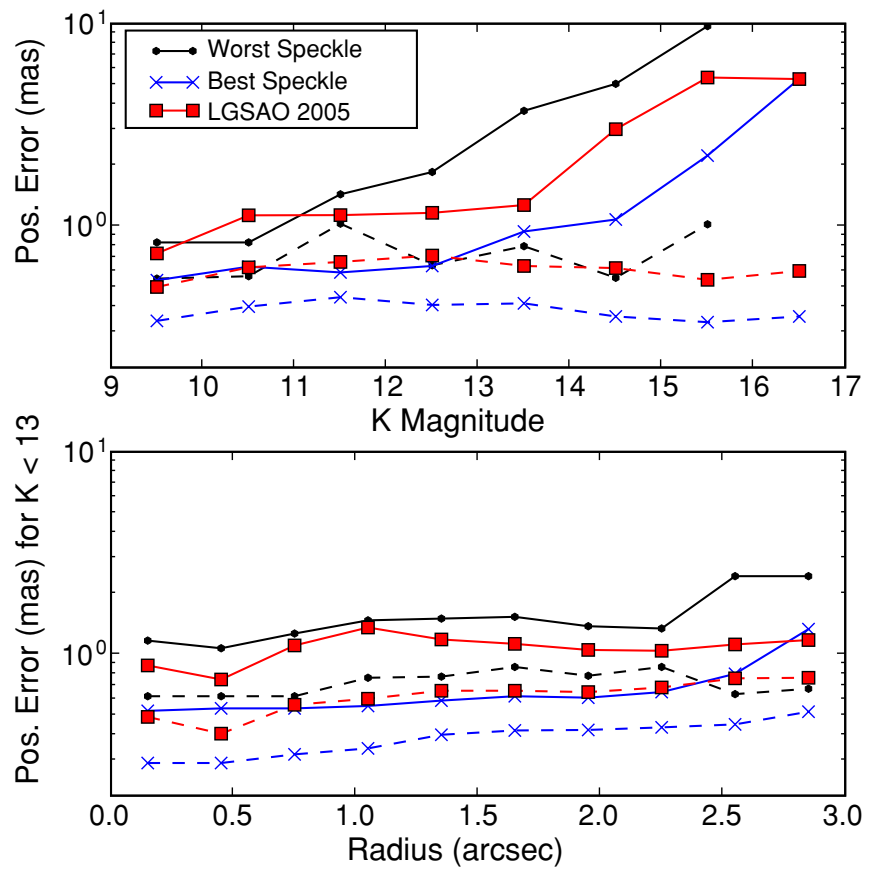

Figure 1. Positional uncertainties for stars as a function of stellar brightness (top) and distance from the black hole, Sgr A*, which is near the center of the field of view (bottom). To show the full range of possible values, the centroiding (solid) and the alignment (dashed) uncertainties are shown for the best (1999.559) and worst (1996.485) speckle epochs and one of the LGS AO epochs (2004.567). The uncertainties are the median values of all stars within magnitude bins of $\Delta K=1$ or radius bins of $\Delta r=0^{\prime \prime} 3$. Note that alignment uncertainties are small compared to centroid uncertainties.

(A color version of this figure is available in the online journal.)

the best-fit values for the free parameters of the transformation, $a_{0}, a_{1}, a_{2}, b_{0}, b_{1}, b_{2}$, for that image. However, after a first pass at the alignment of all the images, proper motions are estimated and used to refine the alignment solutions in a second pass. Sources with estimated proper motions higher than 1.5 mas yr$^{-1}$ $\left(600 \mathrm{~km} \mathrm{~s}^{-1}\right)$ are excluded from the transformation resulting in the elimination of two sources that are near the edge of the speckle field of view and suffer from edge effects. Alignment uncertainties are estimated by a half-sample bootstrap method (Babu \& Feigelson 1996; Ghez et al. 2005a) and are small $\left(\sim 0.2\right.$ mas for stars at $r<2^{\prime \prime}$ ) compared to the centroiding uncertainties (see Figure 1). Alignment and centroiding uncertainties are added in quadrature to produce a final relative positional uncertainty for each star at each epoch. The resulting astrometric data set contain stellar positions and uncertainties for all epochs, transformed into the 2004 July NIRC2 pixel coordinate system $\left(x_{\text {pix }}, y_{\text {pix }}\right)$.

The relative positions and uncertainties are transformed into J2000 absolute astrometric coordinates defined by radio observations of $\mathrm{SiO}$ masers and $\mathrm{Sgr} \mathrm{A}^{*}$. Using observations of the $\mathrm{SiO}$ masers in the infrared, a set of infrared absolute astrometric standards is defined in a process described in detail in an appendix of Ghez et al. (2008). These astrometric standards are used to derive the transformation from 2004 July NIRC2 pixel coordinates into absolute coordinates. A statistically insignificant adjustment is made to place the origin at the dynamical center of S0-2's orbit, which is known to high precision, by offsetting from the radio position of Sgr A* by 1 mas to the east and 5 mas to the south. This offset is well within the absolute astrometric uncertainty of $\sim 6$ mas for Sgr A* (Ghez et al. 2008). The stellar positions in all epochs are 

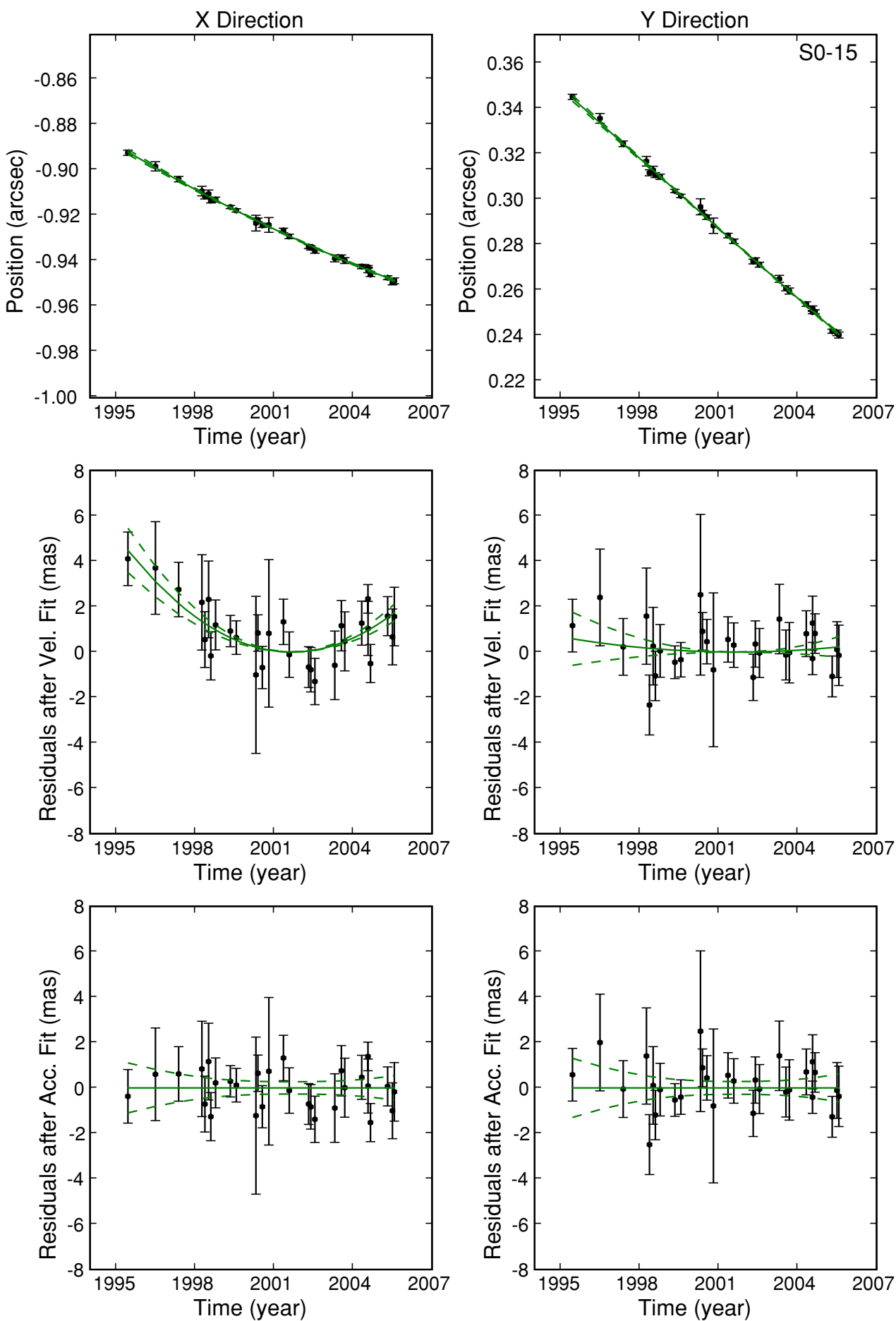

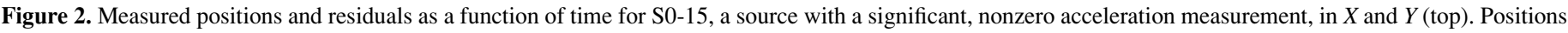

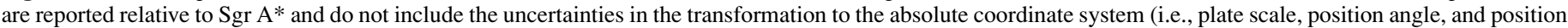

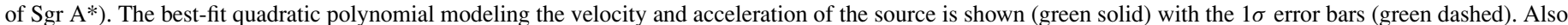

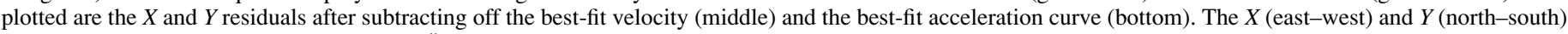
position plots (top) have a ( $y$-axis) range of 0.16 and residual plots (middle, bottom) have a ( $y$-axis) range of \pm 8 mas.

thus expressed in arcseconds offset from the dynamical center with $+x$ increasing east and $+y$ increasing north and can be converted into celestial coordinates using $(x, y)=(\cos \delta \Delta \alpha$, $\Delta \delta) .{ }^{6}$ Positional uncertainties are taken as the quadratic sum of

6 When converting from $(x, y)$ to $(\Delta \alpha, \Delta \delta)$, higher-order terms are negligible $\left(0.06\right.$ mas over $\left.5^{\prime \prime}\right)$ because the celestial sphere is sufficiently flat over our field of view. the relative errors, which dominate, and the absolute error from uncertainties in the plate scale and position angle. Errors in the relative position of $\mathrm{Sgr}^{*}(\sim 2$ mas) are incorporated later during the orbit analysis stage as a parameter of the potential of the supermassive black hole (see Section 4). From the resulting absolute astrometric data set, the sample of young stars is extracted. 

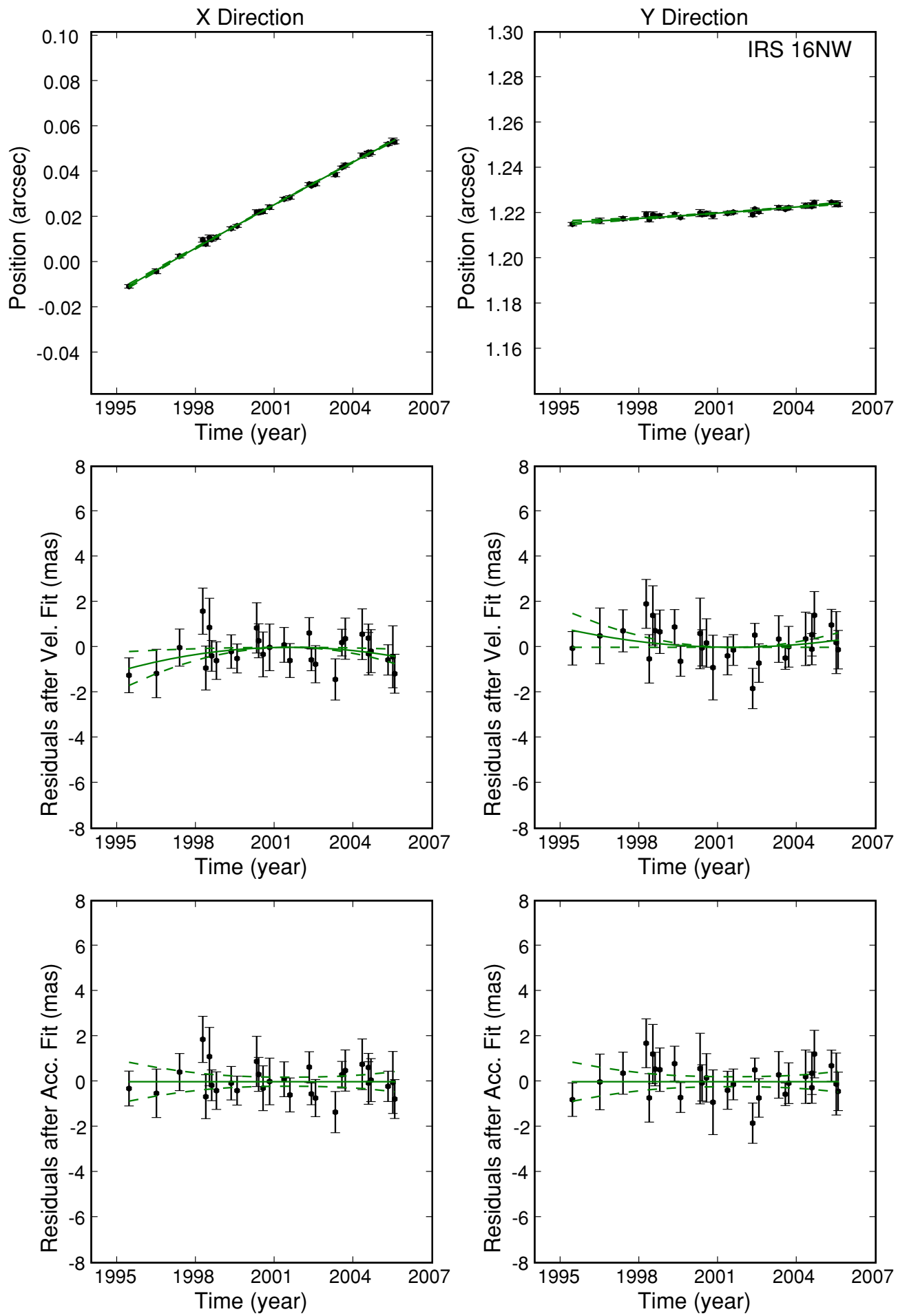

Figure 3. Measured positions and residuals as a function of time for IRS 16NW, a source that has an acceleration consistent with zero, but significantly below the maximum possible acceleration. See the caption in Figure 2 for more information.

\subsection{Proper Motions and Acceleration Results}

For each of the young stars in the sample, positions, velocities, and accelerations in the plane of the sky are derived by fitting second-order polynomials to the star's position as a function of time, weighted by the positional uncertainties. The polynomials are fitted independently in the $x$ - and $y$-coordinates and have the form

$$
x(t)=x_{\text {ref }}+v_{x, \text { ref }}\left(t-t_{\text {ref }}\right)+\frac{1}{2} a_{x, \text { ref }}\left(t-t_{\text {ref }}\right)^{2}
$$

$$
y(t)=y_{\mathrm{ref}}+v_{y, \text { ref }}\left(t-t_{\mathrm{ref}}\right)+\frac{1}{2} a_{y, \text { ref }}\left(t-t_{\mathrm{ref}}\right)^{2},
$$

where $t$ is the time in years, $t_{\text {ref }}$ is a reference time taken to be the mean of the time of all epochs weighted by the positional uncertainties for each star, $x_{\text {ref }}$ and $y_{\text {ref }}$ are the positions at the reference time, $v_{\text {ref }}$ is the velocity at the reference time, and $a_{\text {ref }}$ is the acceleration at the reference time. Uncertainties in the fit parameters are determined from the covariance matrix. Figures 2 and 3 show the polynomial fits for two example stars and the resulting values for the kinematic variables for all stars are 

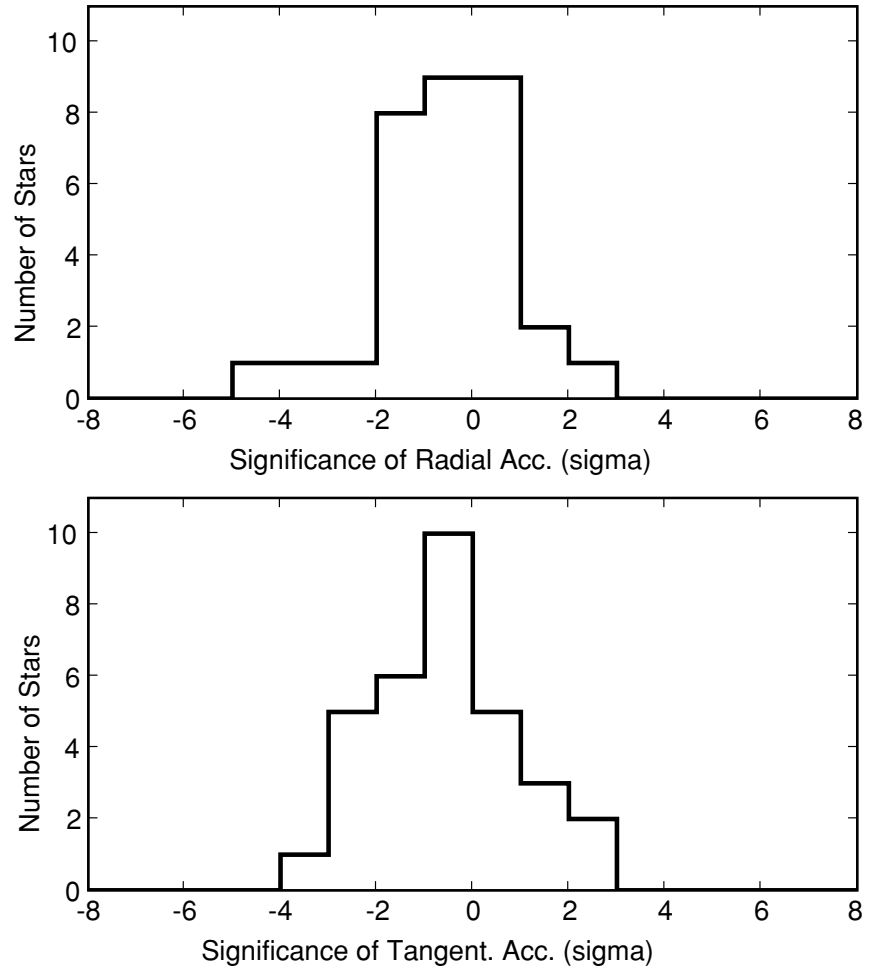

Figure 4. Histograms of $a_{\rho} / \sigma_{a_{\rho}}$ showing the significance of the acceleration measurements in both the radial (top) and tangential (bottom) directions. While, the distributions show an offset from zero indicating a possible bias due to systematic errors, such as residual distortion, that are not well characterized, it appears that any biases are limited to the $\sim 1 \sigma$ level. The only star with significant negative radial acceleration $(\gtrsim 4 \sigma)$ is S0-15 and it is assumed to be a real acceleration due to the gravity of the supermassive black hole.

reported in Table 2. Since the stars' motions are assumed to be dominated by the central force from the black hole, we convert $a_{x, \text { ref }}$ and $a_{y, \text { ref }}$ into radial and tangential accelerations and report only the radial component. ${ }^{7}$ All tangential accelerations and positive radial accelerations are nonphysical and therefore provide a check on the systematic errors of the acceleration measurements. Figure 4 shows a histogram of the significance of the acceleration measurements both in the radial and tangential directions for the young stars in our primary sample. While the tangential and positive radial distributions are slightly offset $(0.6 \sigma)$ from zero and broader $(1.5 \sigma$ versus $1 \sigma)$ than is expected for a normal distribution, any systematic errors appear to impact the results at the $\lesssim 1 \sigma$ level.

The resulting velocity measurements for the young star sample outside the central arcsecond are improved by at least a factor of 7 when compared with our previous work (Ghez et al. 1998; Lu et al. 2005) and other recently reported Galactic center proper motions (e.g., Genzel et al. 2000; Ott 2003). The absolute uncertainties in our proper motions are typically $\sim 0.06$ mas $\mathrm{yr}^{-1}\left(\sim 2 \mathrm{~km} \mathrm{~s}^{-1}\right)$, although stars detected in fewer epochs have somewhat higher values (0.1-0.5 mas $\mathrm{yr}^{-1}$; 4-20 $\left.\mathrm{km} \mathrm{s}^{-1}\right)$. Figures 2 and 3 show examples of the measurements for two stars in our sample (S0-15 and IRS 16NW), and their corresponding proper motion fits with $1 \sigma$ error bars.

In the young star sample, significant $(>3 \sigma)$ acceleration, or curvature, in the plane of the sky is detected only for S0-15

\footnotetext{
7 This assumption may not hold for stars in a gravitationally bound cluster, such as may be the case for the four stars in the extended sample that make up the IRS 13 comoving group; however, the deviations from the potential assumed above should result in only $5 \%-10 \%$ changes in the velocity vectors.
}

(Figure 2). This star has the second smallest projected separation from Sgr $A^{*}$ in our sample, at $\rho=1^{\prime \prime} .0(0.04 \mathrm{pc})$, and has

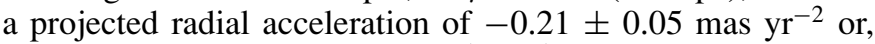
equivalently, $-9.6 \pm 2.0 \mathrm{~km} \mathrm{~s}^{-1} \mathrm{yr}^{-1}$ (see Figure 5). S0-15 is more than twice as far from Sgr $\mathrm{A}^{*}$, in projection, than the seven stars with previously detected accelerations, which were all within a projected radius of less than 0.'4 $(0.016 \mathrm{pc}$; Ghez et al. 2000; Eckart et al. 2002; Ghez et al. 2005a; Eisenhauer et al. 2005).

The detection of acceleration is important in that it allows us to solve for the line-of-sight distance, and thus the threedimensional position of a star relative to the black hole. For a star in the gravitational potential well of a supermassive black hole, the plane-of-the-sky acceleration, at a three-dimensional distance $r$, in cylindrical coordinates is

$$
a_{\rho}=\frac{-G M \rho}{r^{3}}=\frac{-G M \rho}{\left(\rho^{2}+z^{2}\right)^{3 / 2}},
$$

where $\rho$ is the plane-of-the-sky radial coordinate and $z$ is the coordinate along the line of sight relative to Sgr A*. The magnitude of the line-of-sight distance from $\mathrm{Sgr} \mathrm{A}^{*}, z$, can be solved for by adopting a black hole mass of $M_{\bullet}=4.4 \times 10^{6} M_{\odot}$ and a distance of $R_{\circ}=8.0 \mathrm{kpc}$ (see Section 4; Ghez et al. 2008); it is important to note that there is a remaining sign ambiguity for $z$. The resulting line-of-sight distance from Sgr A* for S0-15 is $|0.045 \pm 0.004|$ pc bringing the total separation between $\mathrm{S} 0-15$ and $\mathrm{Sgr} \mathrm{A}^{*}$ to $0.060 \mathrm{pc}$.

The remaining stars in our sample have acceleration measurements that constrain the line-of-sight distance. While the lower limits of these acceleration magnitudes are not significantly different from zero at the $3 \sigma$ level, their upper limits are smaller than the maximum allowed acceleration. The maximum possible magnitude of the acceleration for a star at a given $\rho$ occurs when $z=0$. When the measured acceleration limits are below this value, they provide a lower limit on the star's line-of-sight distance to the SMBH. Figure 6 compares the measured acceleration limits with the maximum possible acceleration for each star. Any $3 \sigma$ acceleration limits below the maximum allowed value gives useful constraints on the line-of-sight distances. In addition to our explicit measurement for S0-15, our high-precision astrometric measurements are now yielding $3 \sigma$ acceleration limits with a median value of -0.19 mas $\mathrm{yr}^{-2}\left(-7.3 \mathrm{~km} \mathrm{~s}^{-1} \mathrm{yr}^{-1}\right)$ that can significantly constrain the line-of-sight distance for nine stars in our sample that are located as far as $1^{\prime \prime} .7(0.07 \mathrm{pc})$, in projection, away from the black hole.

\section{ORBIT ANALYSIS}

For a known point-mass Newtonian gravitational potential, a star's orbital elements can be fully determined from the measurement of only six kinematic variables. For this analysis, we assume that the central point mass is a black hole with characteristics determined by analysis of the orbit of the star S0-2, which has been observed for nearly one complete revolution (Eisenhauer et al. 2005; Ghez et al. 2008). Our proper motion analysis (Section 3.3) yields information on five kinematic variables, including two positions, two velocities, and one acceleration. The sixth kinematic variable comes from radial velocities measured by Paumard et al. (2006). The reported radial velocities are averaged over several years of observations; however, we adopt the same reference epoch, $t_{\text {ref }}$, as for the proper motion analysis since any change in the radial velocity due to acceleration along the line of sight should be well 

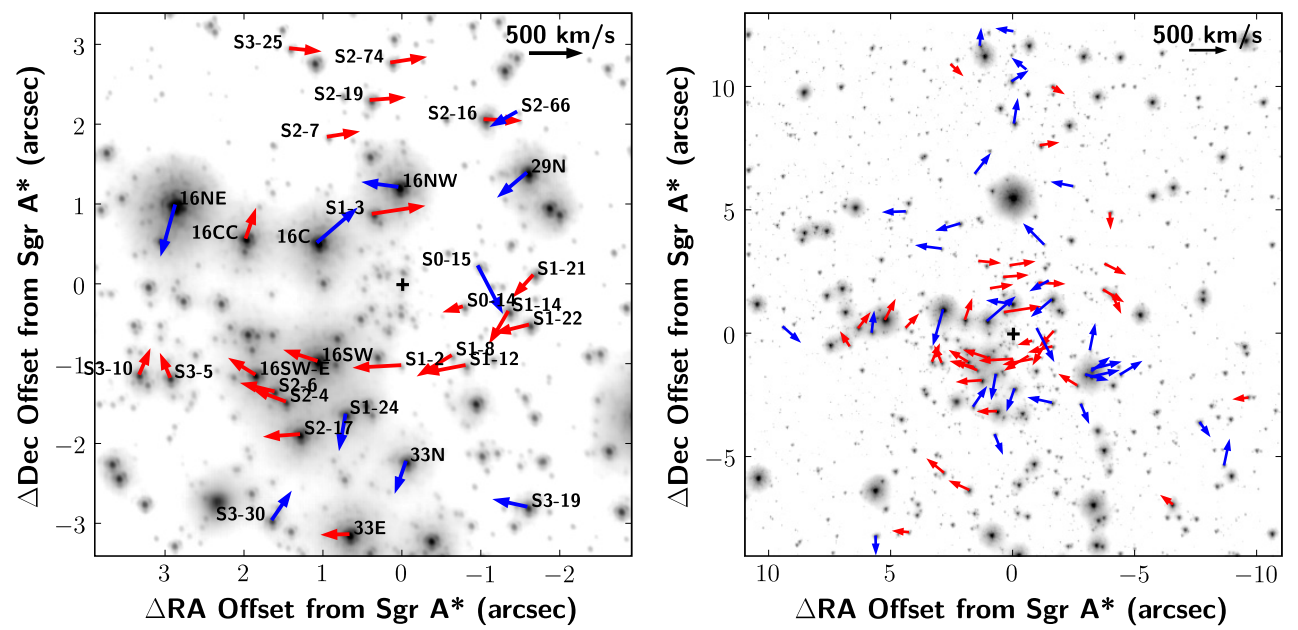

Figure 5. Positions and proper motion vectors of the young stars in our sample. Candidate disk members are shown in red and nondisk members are shown in blue overplotted on an LGS AO image in gray scale. The names of the stars in the primary sample are shown in the left panel and the complete extended sample is shown in a zoomed-out view in the right panel. The position of Sgr A* is marked with a black cross.

Table 2

Proper Motions for Young Stars

\begin{tabular}{|c|c|c|c|c|c|c|c|c|c|c|c|c|}
\hline Name & $\begin{array}{c}K \\
(\mathrm{mag}) \\
\end{array}$ & $N_{\text {epochs }}$ & $\begin{array}{l}\text { Epoch } \\
\text { (year) }\end{array}$ & $\begin{array}{l}\text { Radius } \\
(\operatorname{arcsec})\end{array}$ & $\begin{array}{l}\Delta \text { R.A. }^{\mathrm{a}} \\
(\operatorname{arcsec})\end{array}$ & $\begin{array}{l}\Delta \text { Decl. }^{\mathrm{a}} \\
(\operatorname{arcsec})\end{array}$ & $\begin{array}{c}v_{\text {R.A. }} \\
\left(\mathrm{mas} \mathrm{yr}^{-1}\right)\end{array}$ & $\begin{array}{c}v_{\text {decl. }} \\
\left(\operatorname{mas~yr}^{-1}\right)\end{array}$ & $\begin{array}{c}v_{z}^{\mathrm{b}} \\
\left(\mathrm{km} \mathrm{s}^{-1}\right)\end{array}$ & 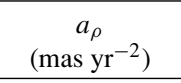 & 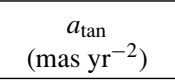 & AltName $^{\mathrm{b}}$ \\
\hline S0-14 & 13.7 & 29 & 2001.290 & 0.82 & -0.770 & -0.270 & $1.62 \pm 0.06$ & $-0.46 \pm 0.07$ & $-14 \pm 40$ & $0.05 \pm 0.05$ & $0.09 \pm 0.05$ & E14 \\
\hline S0-15 & 13.7 & 29 & 2001.680 & 0.97 & -0.930 & 0.280 & $-5.32 \pm 0.07$ & $-10.23 \pm 0.08$ & $-424 \pm 70$ & $-0.21 \pm 0.05$ & $0.10 \pm 0.06$ & E16 \\
\hline $\mathrm{S} 1-3$ & 12.1 & 29 & 2001.980 & 0.98 & 0.440 & 0.879 & $-13.83 \pm 0.05$ & $2.00 \pm 0.05$ & $68 \pm 40$ & $-0.10 \pm 0.03$ & $-0.08 \pm 0.03$ & E15 \\
\hline S1-2 & 14.9 & 26 & 2001.860 & 1.01 & -0.025 & -1.007 & $11.70 \pm 0.13$ & $-0.65 \pm 0.13$ & $26 \pm 30$ & $0.01 \pm 0.08$ & $0.17 \pm 0.08$ & E17 \\
\hline $\mathrm{S} 1-8$ & 14.2 & 29 & 2001.680 & 1.08 & -0.651 & -0.865 & $7.64 \pm 0.10$ & $-4.63 \pm 0.10$ & $-364 \pm 40$ & $0.04 \pm 0.06$ & $0.06 \pm 0.06$ & E18 \\
\hline IRS 16NW & 10.1 & 29 & 2001.560 & 1.22 & 0.029 & 1.221 & $6.30 \pm 0.06$ & $0.87 \pm 0.06$ & $-44 \pm 20$ & $0.04 \pm 0.04$ & $-0.05 \pm 0.04$ & E19 \\
\hline IRS 16C & 9.8 & 29 & 2001.570 & 1.23 & 1.121 & 0.497 & $-8.74 \pm 0.05$ & $7.42 \pm 0.05$ & $125 \pm 30$ & $-0.04 \pm 0.03$ & $-0.11 \pm 0.03$ & E20 \\
\hline $\mathrm{S} 1-12$ & 13.8 & 28 & 2001.500 & 1.30 & -0.837 & -1.000 & $9.93 \pm 0.07$ & $-1.88 \pm 0.07$ & $-24 \pm 30$ & $0.04 \pm 0.06$ & $-0.00 \pm 0.06$ & E21 \\
\hline $\mathrm{S} 1-14$ & 12.8 & 29 & 2001.380 & 1.39 & -1.355 & -0.302 & $4.01 \pm 0.06$ & $-6.79 \pm 0.07$ & $-434 \pm 50$ & $-0.11 \pm 0.05$ & $-0.02 \pm 0.05$ & E22 \\
\hline IRS 16SW & 10.0 & 29 & 2001.490 & 1.43 & 1.051 & -0.966 & $6.80 \pm 0.05$ & $2.22 \pm 0.06$ & $320 \pm 40$ & $-0.08 \pm 0.04$ & $0.03 \pm 0.04$ & E23 \\
\hline $\mathrm{S} 1-21$ & 13.3 & 17 & 2001.190 & 1.68 & -1.669 & 0.141 & $3.52 \pm 0.09$ & $-3.84 \pm 0.09$ & $-344 \pm 50$ & $0.03 \pm 0.07$ & $0.02 \pm 0.07$ & E24 \\
\hline $\mathrm{S} 1-22$ & 12.7 & 29 & 2001.200 & 1.70 & -1.631 & -0.493 & $6.95 \pm 0.07$ & $-1.70 \pm 0.08$ & $-224 \pm 50$ & $-0.09 \pm 0.06$ & $-0.08 \pm 0.06$ & E25 \\
\hline $\mathrm{S} 1-24$ & 11.6 & 29 & 2001.420 & 1.75 & 0.718 & -1.591 & $1.13 \pm 0.07$ & $-6.37 \pm 0.08$ & $206 \pm 30$ & $0.02 \pm 0.05$ & $0.08 \pm 0.05$ & E26 \\
\hline S2-4 & 12.3 & 29 & 2001.480 & 2.07 & 1.452 & -1.476 & $6.69 \pm 0.08$ & $2.51 \pm 0.08$ & $286 \pm 20$ & $-0.03 \pm 0.06$ & $-0.06 \pm 0.06$ & E28 \\
\hline IRS $16 \mathrm{CC}$ & 10.6 & 25 & 2000.840 & 2.07 & 1.999 & 0.550 & $-1.88 \pm 0.06$ & $5.48 \pm 0.06$ & $241 \pm 25$ & $-0.02 \pm 0.04$ & $-0.09 \pm 0.04$ & E27 \\
\hline S2-6 & 12.1 & 29 & 2001.290 & 2.09 & 1.594 & -1.345 & $6.80 \pm 0.05$ & $1.66 \pm 0.06$ & $216 \pm 20$ & $-0.02 \pm 0.04$ & $-0.02 \pm 0.04$ & E30 \\
\hline S2-7 & 14.1 & 27 & 2002.350 & 2.09 & 0.979 & 1.849 & $-6.15 \pm 0.11$ & $1.01 \pm 0.11$ & $-94 \pm 50$ & $0.04 \pm 0.08$ & $-0.07 \pm 0.08$ & E29 \\
\hline IRS $29 \mathrm{~N}$ & 10.3 & 29 & 2001.410 & 2.14 & -1.595 & 1.423 & $5.26 \pm 0.08$ & $-4.41 \pm 0.08$ & $-190 \pm 90$ & $-0.02 \pm 0.06$ & $-0.09 \pm 0.06$ & E31 \\
\hline IRS 16SW-E & 11.0 & 29 & 2001.430 & 2.17 & 1.846 & -1.141 & $4.83 \pm 0.06$ & $2.98 \pm 0.06$ & $366 \pm 70$ & $-0.03 \pm 0.04$ & $-0.03 \pm 0.04$ & E32 \\
\hline IRS 33N & 11.4 & 29 & 2001.630 & 2.19 & -0.048 & -2.189 & $1.72 \pm 0.12$ & $-5.15 \pm 0.12$ & $68 \pm 20$ & $-0.01 \pm 0.08$ & $0.18 \pm 0.08$ & E33 \\
\hline S2-17 & 10.9 & 29 & 2001.660 & 2.26 & 1.271 & -1.871 & $7.51 \pm 0.09$ & $-0.51 \pm 0.09$ & $100 \pm 20$ & $-0.07 \pm 0.07$ & $0.04 \pm 0.07$ & E34 \\
\hline $\mathrm{S} 2-16$ & 11.9 & 29 & 2001.410 & 2.30 & -0.992 & 2.073 & $-8.07 \pm 0.08$ & $-0.29 \pm 0.09$ & $-100 \pm 70$ & $0.08 \pm 0.06$ & $-0.01 \pm 0.06$ & E35 \\
\hline S2-19 & 12.6 & 28 & 2001.770 & 2.35 & 0.446 & 2.310 & $-7.30 \pm 0.09$ & $0.55 \pm 0.09$ & $41 \pm 20$ & $0.02 \pm 0.06$ & $-0.05 \pm 0.06$ & E36 \\
\hline S2-66 & 14.8 & 21 & 2003.490 & 2.62 & -1.457 & 2.173 & $3.25 \pm 0.46$ & $-1.57 \pm 0.46$ & $-114 \pm 30$ & $0.64 \pm 0.22$ & $-0.18 \pm 0.22$ & E37 \\
\hline $\mathrm{S} 2-74$ & 13.3 & 24 & 2002.670 & 2.78 & 0.179 & 2.779 & $-7.63 \pm 0.17$ & $1.14 \pm 0.17$ & $36 \pm 20$ & $-0.01 \pm 0.09$ & $-0.08 \pm 0.09$ & E38 \\
\hline IRS 16NE & 9.0 & 28 & 2000.990 & 3.06 & 2.868 & 1.053 & $3.11 \pm 0.06$ & $-10.94 \pm 0.06$ & $-10 \pm 20$ & $-0.06 \pm 0.04$ & $-0.11 \pm 0.04$ & E39 \\
\hline S3-5 & 12.2 & 29 & 2001.030 & 3.17 & 2.938 & -1.183 & $1.44 \pm 0.08$ & $3.44 \pm 0.08$ & $327 \pm 100$ & $-0.01 \pm 0.06$ & $-0.13 \pm 0.06$ & $\mathrm{E} 40$ \\
\hline IRS 33E & 10.6 & 16 & 2003.890 & 3.20 & 0.665 & -3.126 & $5.38 \pm 0.49$ & $0.04 \pm 0.50$ & $170 \pm 20$ & $0.02 \pm 0.18$ & $-0.36 \pm 0.18$ & E41 \\
\hline S3-19 & 12.5 & 17 & 2003.700 & 3.21 & -1.591 & -2.785 & $6.40 \pm 0.49$ & $1.65 \pm 0.50$ & $-114 \pm 50$ & $-0.28 \pm 0.19$ & $-0.15 \pm 0.18$ & E43 \\
\hline S3-25 & 14.1 & 18 & 2003.030 & 3.30 & 1.452 & 2.963 & $-5.86 \pm 0.35$ & $-0.84 \pm 0.37$ & $-114 \pm 40$ & $-0.12 \pm 0.14$ & $0.06 \pm 0.14$ & E44 \\
\hline S3-30 & 12.9 & 25 & 2003.120 & 3.40 & 1.668 & -2.963 & $-3.14 \pm 0.27$ & $5.07 \pm 0.28$ & $91 \pm 30$ & $-0.21 \pm 0.19$ & $-0.27 \pm 0.18$ & E47 \\
\hline S3-10 & 12.4 & 26 & 2001.780 & 3.54 & 3.345 & -1.143 & $-1.78 \pm 0.12$ & $4.11 \pm 0.13$ & $281 \pm 20$ & $-0.14 \pm 0.08$ & $-0.17 \pm 0.09$ & E50 \\
\hline
\end{tabular}

Notes. All uncertainties are $1 \sigma$ relative errors and do not include errors in the plate scale, location of Sgr A*, or position angle.

${ }^{\text {a }}$ Positions as determined from polynomial fitting have relative errors of $\sim 0.4$ mas.

${ }^{b}$ Radial velocities and alternate names obtained from Paumard et al. (2006).

within the large measurement uncertainties in radial velocity $\left(\sigma_{v_{z, \text { ref }}} \sim 20-100 \mathrm{~km} \mathrm{~s}^{-1}\right)$. As described in Section 3.3, the plane-of-the-sky acceleration can be converted into a line-ofsight distance that, when combined with the projected distance, gives the full three-dimensional position for a star. Although most of the stars in our sample have plane-of-the-sky accelerations that are consistent with zero, the upper limits on the magnitude of the acceleration provide valuable information by ruling out small line-of-sight distances. We therefore use our best-fit accelerations and uncertainties as formal measures of 


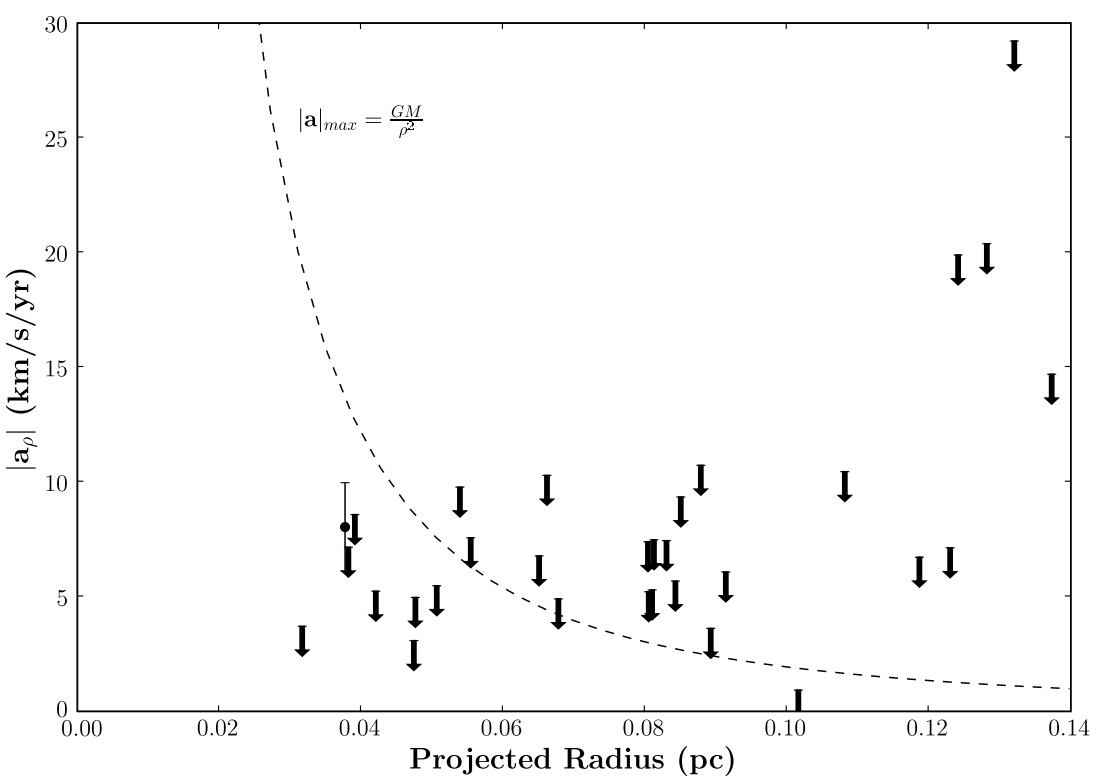

Figure 6. Significance of observed limits for the plane-of-the-sky acceleration. For a given projected radius, there is a maximum allowed acceleration (dashed line). If the measured accelerations from polynomial fitting, shown as $3 \sigma$ upper limits on the $y$-axis, are less than the maximum allowed acceleration (below the dashed line), then significant constraints can be placed on the line-of-sight distance, $z$, and subsequently the orbital parameters of the star. S0-15 has a significant detection of nonzero acceleration and is plotted with its $1 \sigma$ error bars.

the acceleration when converting to a line-of-sight distance. Therefore, the six measured quantities can be expressed as a three-dimensional position and three-dimensional velocity at a certain epoch $\left(t_{\text {ref }}\right)$. Given the properties of the black hole, these kinematic quantities can be translated directly into six standard orbital elements (see Appendix B).

A Monte Carlo simulation is carried out to transform each star's six measured kinematic variables $\left(x_{\text {ref }}, y_{\text {ref }}, v_{x, \text { ref }}, v_{y \text {,ref }}\right.$, $\left.v_{z, \text { ref }}, a_{\rho, \text { ref }}\right)$ into six orbital parameters $\left(i, \Omega, \omega, e, P, T_{o}\right)$ and their uncertainties. A total of $10^{5}$ Monte Carlo trials are run and, in each trial, $4+(6 \cdot 32)$ variables are randomly generated; four for the potential parameters and six for each of the 32 stars' measured kinematic variables. The four potential parameters are pulled from a four-dimensional probability density function, $\operatorname{PDF}\left(M_{\bullet}, R_{o}, x_{o}, y_{o}\right)$, based on the orbit of S0-2 derived by Ghez et al. (2008), where the black hole's mass and line-of-sight distance are centered on $M_{\bullet}=4.4 \times 10^{6} M_{\odot}$ and $R_{\circ}=8.0 \mathrm{kpc}{ }^{8}$ the dynamical center is adopted as the origin with $x_{o}$ and $y_{o}$ defined as zero, and the projected one-dimensional probability distributions' rms errors are $[1.0,1.6]$ mas for $\left[x_{o}, y_{o}\right], 0.3 \times$ $10^{6} M_{\odot}$ for $M_{\bullet}$, and $0.3 \mathrm{kpc}$ for $R_{\circ} .{ }^{9}$ For each trial, all the stars' orbits are calculated using the same potential parameters in order to preserve correlations between the potential parameters and the orbital parameters such as eccentricity. The kinematic variables for each star are sampled from independent Gaussian distributions, each of which is centered at the best-fit value from Table 2 and has a $1 \sigma$ width set to the measurement uncertainty. Any correlations between the measured kinematic variables are negligible given the small uncertainties in the stars' relative angular positions $(\lesssim 0.2 \%)$ and velocities $(\lesssim 3 \%)$ in the planeof-the-sky as compared to the uncertainties in the black hole

\footnotetext{
8 These values correspond to a 12-parameter orbit model for S0-2 (i.e., $v_{z}=0$ case) from an early version of Ghez et al. (2008). In this version, local distortions were not corrected (Ghez et al. 2008, Appendix B); but the resulting black hole mass and distance differ by $<1 \sigma$ from the final reported values.

9 Simulations were also performed using the lower black hole mass and distance reported by (Eisenhauer et al. 2005). Our results on the detection of only one stellar disk and on the properties of the disk are all consistent within $1 \sigma$ error bars.
}

mass $(\sim 10 \%)$ and the accelerations $(\sim 60 \%)$. The distribution for the acceleration, $a_{\rho}$, is truncated such that only accelerations of bound orbits are allowed, ${ }^{10}$ which follows from requiring a negative specific orbital energy,

$$
E=\frac{v^{2}}{2}-\frac{G M}{r}<0
$$

and substituting from Equation (5) to give the acceleration constraint

$$
\left|a_{\rho}\right|>\frac{\rho v^{6}}{8(G M)^{2}} .
$$

For each trial and each star, the orbital parameters are computed and the results of all the trials are combined into a sixdimensional probability density function (PDF) by dividing up parameter space into bins, summing the number of trials in each bin, and then normalizing by the total number of trials. This Monte Carlo method is a straightforward way to combine a star's six measurement PDFs and the four-dimensional PDF for the central point mass, which shows strong correlations between $M$. and $R_{o}$, to produce a six-dimensional PDF for each star's orbital elements, $\operatorname{PDF}\left(i, \Omega, \omega, e, P, T_{o}\right)$, which has strong correlations between the orbital parameters. The results of these simulations are plotted for an example star, IRS 16SW, in Figure 7 to show that $i$ and $\Omega$ are generally well determined and that $e$, in some cases, can be usefully constrained. Similar figures of the orbital parameters for every star are shown in Figure set 7, which is available online in the electronic edition of this paper.

The resulting stellar orbital parameters are constrained by several different factors. First, a measured acceleration that is significantly different from zero, such as for $\mathrm{S} 0-15$, yields the best-determined orbit since the line-of-sight distance is confined

\footnotetext{
10 The assumption that the orbits are bound does not affect the results presented in this paper discussed in Section 5 since all unbound orbital solutions yield high inclination (edge-on) orbits and large eccentricities $(e>1)$. Considering only bound orbits simplifies the orbit analysis as we need only consider equations for elliptical orbits rather than hyperbolic or parabolic orbits.
} 

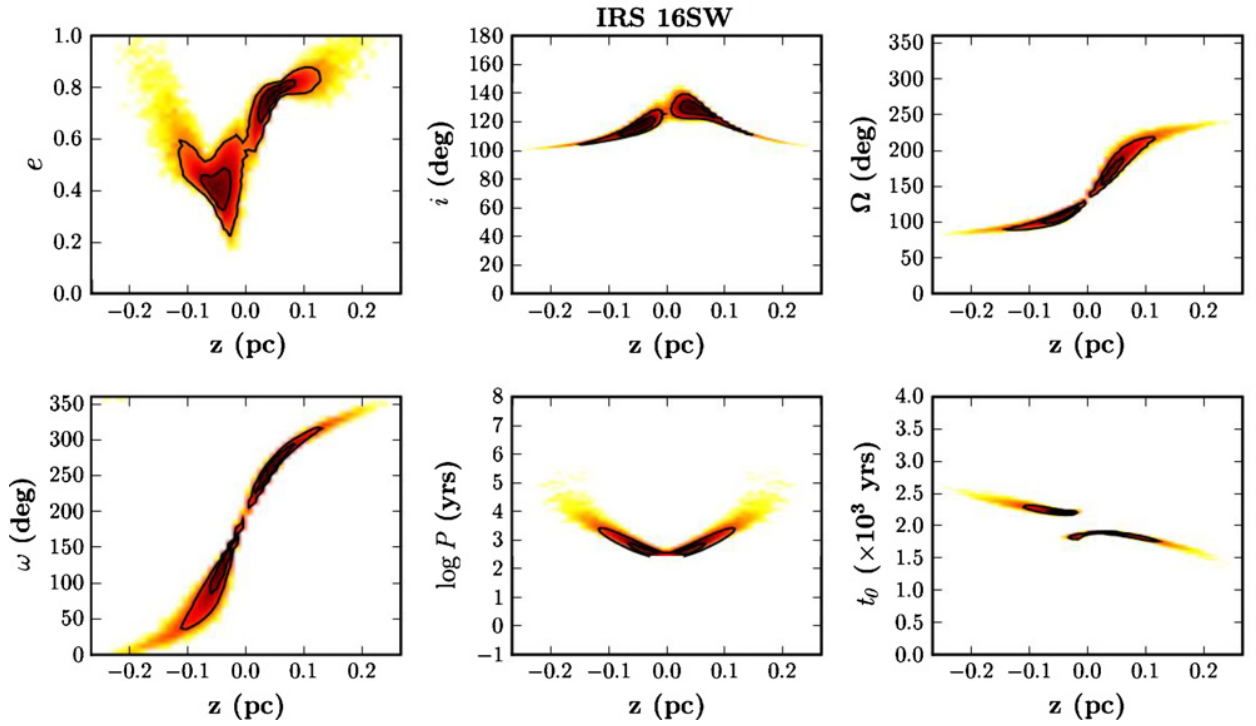

Figure 7. Range of allowed orbital parameters for IRS 16SW as determined from the observed two-dimensional position in the plane of the sky, the three-dimensional velocity, and the acceleration. The probability distribution for each orbital parameter is determined by sampling from a Gaussian distribution for each of the observed quantities and analytically converting to the standard orbital elements. High density (dark) regions represent the most probable values for each orbital parameter and the resulting $1 \sigma$ and $2 \sigma$ contours are shown as black lines.

(An extended figure set is available in the online journal.)
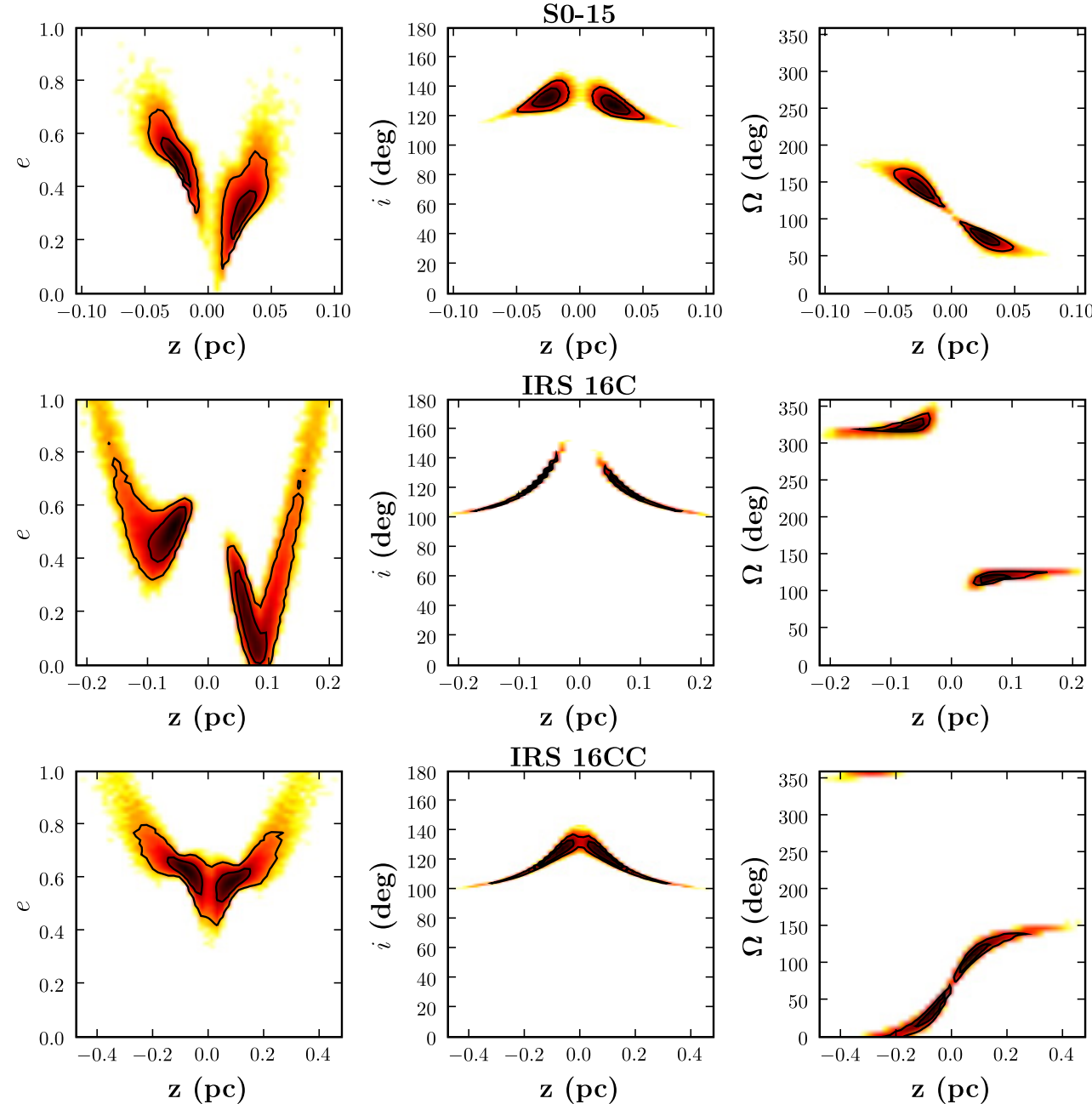

Figure 8. Range of allowed eccentricities $(e)$, inclinations $(i)$, and angles to ascending nodes $(\Omega)$ as determined by our orbit analysis for three example stars. The range of $z$ values (horizontal axis) extends to all possible bound orbits for the star. The PDF is shown in color with the $1 \sigma$ and $2 \sigma$ contours drawn as black lines. S0-15 has a measured acceleration that is significantly different from zero. IRS 16C has an acceleration upper limit that is less than the maximum allowed acceleration, and thus a lower limit on the line-of-sight distance, $|z|$. IRS $16 \mathrm{CC}$ has no significant acceleration limit, but has a high velocity that is always larger than the circular velocity, thus prohibiting circular orbits. Also, by assuming the star is bound, the direction of the normal vector to IRS 16CC's orbital plane is restricted to a low inclination. 
to a small range of values (Figure 8, top). Secondly, each star has a maximum allowed acceleration, $a_{\rho, \max }=\left|-G M / \rho^{2}\right|$, at the closest possible distance set by the observed projected radius. Stars with measured accelerations more than $3 \sigma$ below the maximum allowed acceleration, such as IRS $16 \mathrm{NW}$, have strong lower limits on their line-of-sight distances, which translate into significant constraints on the direction of the angular momentum vector, $\vec{L}$, and can be equivalently expressed as constraints on inclination, $i$, and on the angle to the ascending node, $\Omega$ (Figure 8, middle). Finally, even stars without significant limits on their line-of-sight distance from accelerations have some well-constrained orbital elements. In particular, $i$ and $\Omega$ are well constrained as a result of the precise measurements for the stellar velocities and potential parameters. Furthermore, if the star's total velocity is higher than the circular velocity at the twodimensional projected radius, then it is higher than the circular velocity at all distances and only nonzero eccentricity orbits are allowed (Figure 8, bottom).

The Monte Carlo analysis described above assumes that, in the absence of an acceleration measurement, the acceleration should be drawn from a uniform probability distribution; or, in other words, we adopt a uniform acceleration prior. For those stars that are only in the extended sample, the Monte Carlo orbit analysis samples from this uniform acceleration prior ranging from the largest allowed acceleration by the projected radius to the smallest allowed for the orbit to remain bound. For these stars and for stars in the primary sample with acceleration limits that are not significantly smaller than the maximum physically allowed acceleration, the uniform- $a_{\rho}$ prior is an important assumption. To test how sensitive our results are to this assumption, we performed the same Monte Carlo analysis as detailed above using an alternative assumption that the prior acceleration distribution is uniform in $z$, which shifts the line-of-sight distance PDF to larger values when compared with a uniform- $a_{\rho}$ prior. On a star-by-star basis, the resulting orbital parameters are consistent within $1 \sigma$ for both priors, with one exception. The young star $\mathrm{S} 0-14$ has an eccentricity that is constrained to be higher than $0.93(3 \sigma)$ with a uniform- $a_{\rho}$ prior, while with a uniform- $z$ prior, all eccentricities are allowed within $3 \sigma$. S0-14 is distinguishable from all other stars in our sample in that it has a total velocity of only $50 \mathrm{~km} \mathrm{~s}^{-1}$, as compared to $160-640 \mathrm{~km} \mathrm{~s}^{-1}$ for the rest of the sample. Such a small velocity translates into a very large range of allowed lineof-sight distances which are not well sampled by a uniform$a_{\rho}$ prior. S0-14's ranges of $i$ and $\Omega$ are not largely affected by the choice of prior; therefore, we exclude $\mathrm{S} 0-14$ from our eccentricity analysis, but we keep it in all other orbital analyses.

To distinguish between these two possible priors, we examine the resulting distribution of orbital phases. For a set of stars whose motion is dominated by the supermassive black hole and that have been orbiting for more than a few orbital time scales, the distribution of orbital phases should be uniform. The distribution of orbital phases for our sample is constructed by summing the orbital phase PDFs for all the stars. Figure 9 shows that while the uniform- $a_{\rho}$ prior produces a population that is uniformly distributed in orbital phase, the uniform- $z$ prior produces a distribution that is strongly peaked at 0 (periapse) due to the higher occurrence of large line-of-sight distances that, for a given velocity, creates an artificial bias toward periapse. Such a strong bias toward periapse is unlikely to occur even if some of the young stars reside in a gravitationally bound cluster, such as IRS 13, where all the cluster members would have a similar orbital phase. Based on our assumption that the

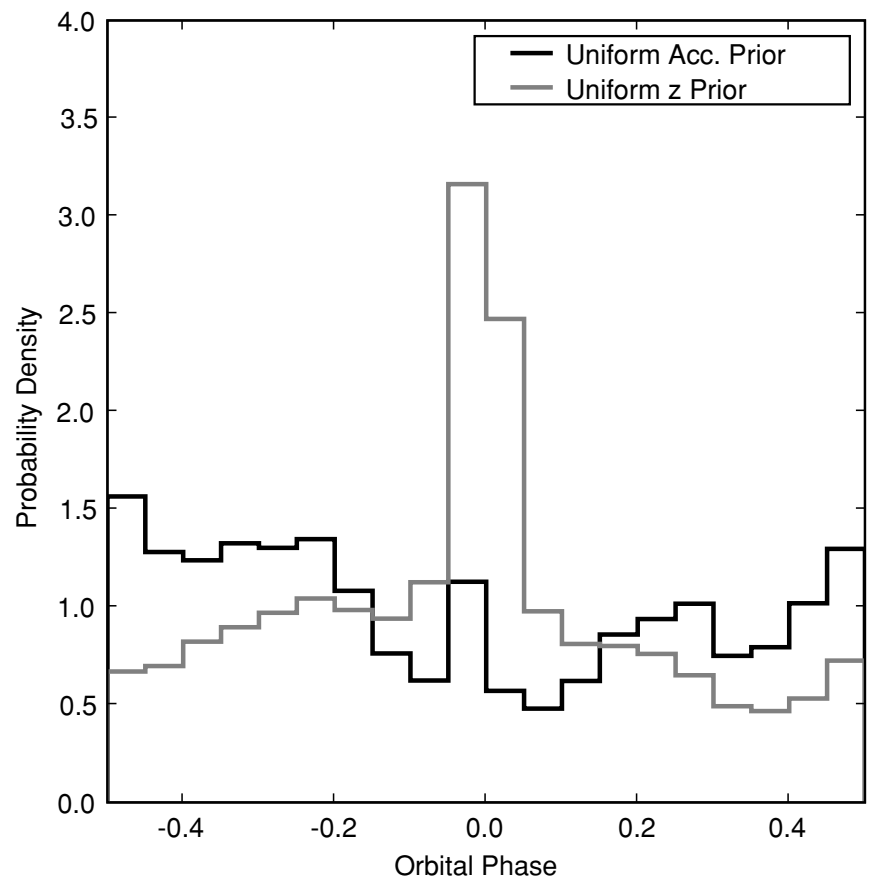

Figure 9. Resulting distribution of orbital phases for all stars when assuming either a uniform acceleration prior (black) or a uniform $z$ prior (gray) and then imposing the measured accelerations. The uniform $z$ prior shows a strong bias toward an orbital phase of 0 , which corresponds to periapse; while the uniform acceleration prior shows a more uniform distribution.

distribution of orbital phases should be roughly uniform, we adopt a uniform- $a_{\rho}$ prior instead of the uniform- $z$ prior in the following sections.

\section{ORBIT RESULTS}

\subsection{Detection of the Clockwise Disk}

A large number of stars appear to share a common orbital plane based on our analysis, which has no prior assumption about the existence of a disk. The orientation of a star's orbital plane can be described by a unit vector originating at Sgr A*'s position and pointing normal to the orbital plane $(\vec{n})$; and this normal vector's direction can be expressed by the inclination angle $(i)$ and the angle to the ascending node $(\Omega)$ using

$$
\vec{n}=\left(\begin{array}{l}
n_{x} \\
n_{y} \\
n_{z}
\end{array}\right)=\left(\begin{array}{cc}
\sin i & \cos \Omega \\
-\sin i & \sin \Omega \\
-\cos i
\end{array}\right) .
$$

The direction of each star's orbital plane normal vector is determined from the joint two-dimensional PDF of $i$ and $\Omega$, $\operatorname{PDF}(i, \Omega)$, which is constructed by binning the resulting $i$ and $\Omega$ values from the Monte Carlo simulation in a two-dimensional histogram with equal solid angle bins using the HEALpix framework (Górski et al. 2005). Figure 10 shows $\operatorname{PDF}(i, \Omega)$ projected onto the sky as viewed from $\mathrm{Sgr} \mathrm{A}^{*}$ for the same three example stars shown in Figure 8. Figure 11 shows, for all stars, the contours for the $68 \%$ confidence region, which, on average, covers a solid angle of $\mathrm{SA}_{\vec{n}} \sim 0.2$ steradian (sr) for the primary sample and $0.6 \mathrm{sr}$ for stars found only in the extended sample, which have larger proper motion uncertainties. Tables 3 and 4 list this solid angle, $\mathrm{SA}_{\vec{n}}$, for each star in the primary and extended samples. The bound orbit assumption does not greatly impact the size of the $\mathrm{SA}_{\vec{n}}$ because the orbital parameters $i$ and $\Omega$ asymptote at large line-of-sight distances as can be seen 

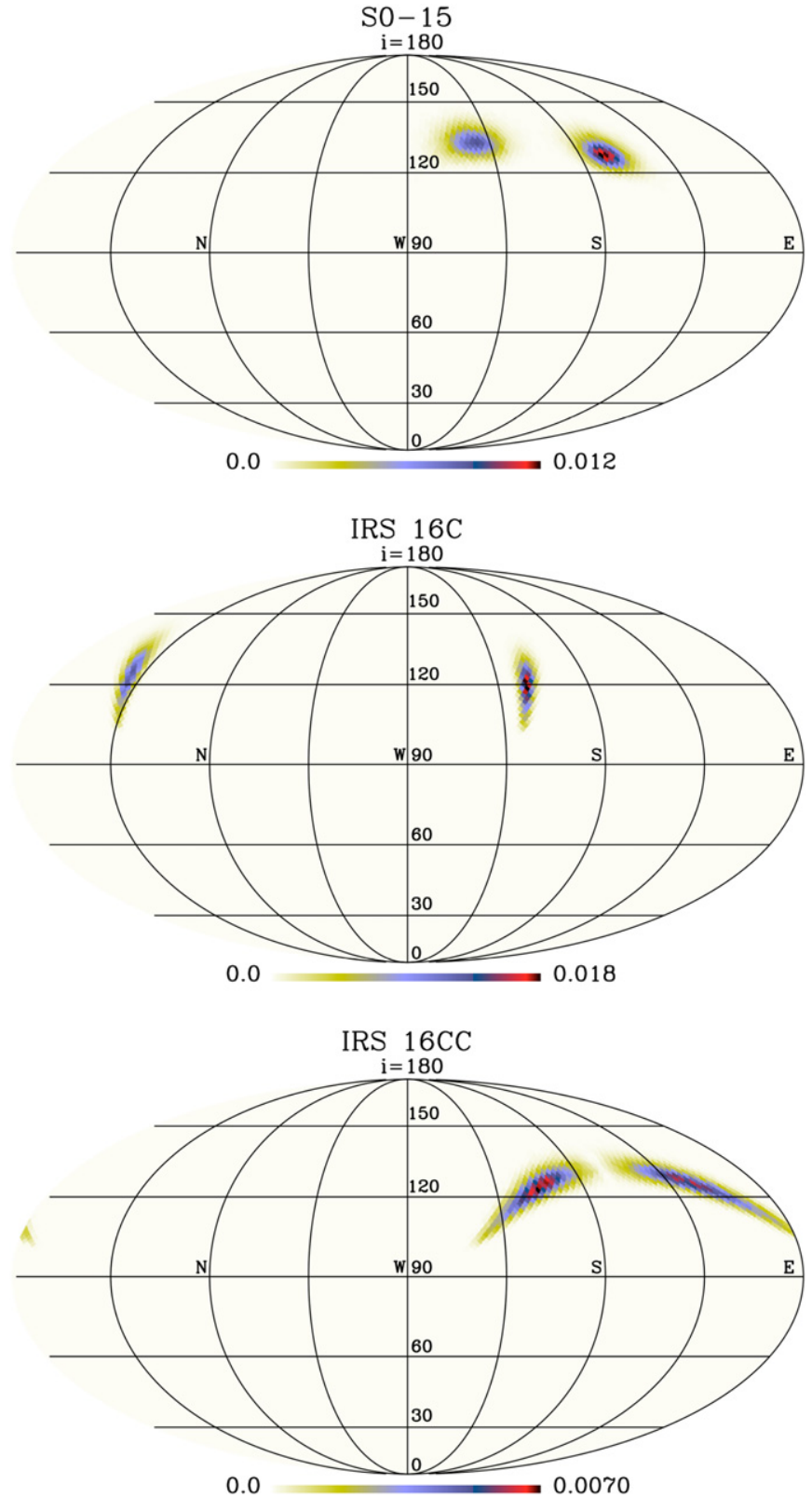

Figure 10. Orientation of three stars' orbital planes as described by the probability distribution of the planes' normal vector projected onto the sky as viewed from Sgr A*. Colors indicate the probability density for a star's normal vector to point at each pixel on the sky. The constraint on the stars' normal vectors are set by (top: S0-15) a measured acceleration; (middle: IRS 16C) a significant acceleration limit; (bottom: IRS 16CC) the star's high velocity and assuming the orbit is bound.

in Figure 8. Stars with acceleration limits significantly smaller than $a_{\rho \text {,max }}$ have two isolated solutions because small line-ofsight distances $(z)$ are not permitted and at large line-of-sight distances the positive- $z$ and negative- $z$ solutions asymptote to two different values of $\Omega$ (see Figure 8 ). Despite this degeneracy, the clockwise $\left(i=90^{\circ}-180^{\circ}\right)$ stars' normal vectors appear to cluster around a common point indicating that many of these stars lie on a common orbital plane.

The directions of the stars' normal vectors show a statistically significant clustering as measured by the density of normal vectors in the sky as viewed from Sgr $\mathrm{A}^{*}$. To quantify the density of normal vector directions, we use a nearest neighbor density estimate, which is commonly used to identify galaxy clusters (e.g., Dressler 1980), and take the density at each point on the sky to be

$$
\Sigma=\frac{k}{2 \pi\left(1-\cos \theta_{k}\right)} \text { stars } \mathrm{sr}^{-1},
$$

where $\theta_{k}$ is the angle to the $k$ th nearest star and $k$ is taken to be 6 . We calculate the expectation value for the density of normal vectors at each point on the sky using the Monte Carlo simulation discussed earlier. For each Monte Carlo trial, the sky is divided into 12,288 equal area pixels $(0.001 \mathrm{sr})$ using a HEALpix grid and the density of normal vectors is calculated for each pixel. These estimates are then averaged together over all the trials to provide an average density per pixel on the sky. The resulting average density of normal vectors is nearly the same for a choice of 4th, 5th, or 7th nearest neighbor. Additionally, a similar analysis using a fixed aperture to calculate the density of normal vectors at each point on the sky produced similar, but less smooth, results as the nearest neighbor approach we adopt here. A peak in the density of normal vectors is detected at $i=115^{\circ} \pm 3^{\circ}$ and $\Omega=100^{\circ} \pm 3^{\circ}$, which provides direct evidence of a common orbital plane without any prior assumptions (see Figure 12). The uncertainty on the peak position is taken as the half-width at half-maximum of the peak divided by the square root of the number of stars that are candidate disk members, $\sqrt{N_{\text {disk-stars }}}$ (see below). We also note that an analysis of the entire extended sample produces a peak at exactly the same position. The mean density of normal vectors at the peak is 0.016 stars $\mathrm{deg}^{-2}$ with a negligible uncertainty on the mean value $\left(<10^{-4}\right.$ stars $\left.\mathrm{deg}^{-2}\right)$. The significance of the peak is determined by comparing the background density of normal vectors, which is defined by the

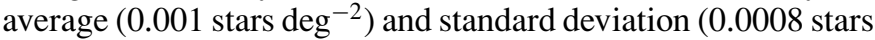
$\mathrm{deg}^{-2}$ ) of all other pixels on the sky after first rejecting those pixels $(\sim 0.25 \mathrm{sr})$ that are high outliers (more than three standard deviations). The density peak is $\sim 19 \sigma$ above the observed background density. A second comparison can be made to the density expected if the 32 stars in our sample were isotropically distributed over $4 \pi$ steradians. The observed peak in the density is $\gtrsim 20$ times higher than this isotropic density. Thus, we conclude that there is a statistically significant common orbital plane of young stars.

The majority of the young stars that are orbiting in the clockwise direction are likely to be orbiting in this common plane. A comparison of each star's normal vector to the common plane's normal vector allows us to determine which stars are not on the common plane with high statistical significance. All other stars are then considered candidate members. First, a preliminary estimate of the thickness of the common plane is determined by defining the solid angle extent of the plane,

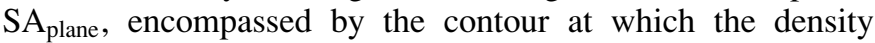
drops to half of the peak value. This corresponds to a region with a solid angle of $\mathrm{SA}_{\text {plane }} \sim 0.1 \mathrm{sr}$, which gives a halfopening angle of 0.2 radians $\left(10^{\circ}\right)$ for a cone with the same $\mathrm{SA}_{\text {plane }}$. Then, each star's $\operatorname{PDF}(i, \Omega)$, is integrated over this region to determine the probability that the star is a disk member. The orientation of the stars' normal vectors has a wide range of uncertainties as expressed by the total solid angle covered by each star, so it is necessary to distinguish between those stars that have a low probability due to a large $\vec{n}$-uncertainty (i.e., large solid angle) versus those stars that have a low probability because they are significantly offset from the common plane. Therefore, we normalize the above integrated probability by the probability at the peak of the star's PDF 
Table 3

Orbital Eccentricity and Disk Membership

\begin{tabular}{|c|c|c|c|c|c|c|c|}
\hline \multirow[t]{2}{*}{ Name } & \multirow{2}{*}{$\begin{array}{c}\mathrm{SA}_{\vec{n}} \\
\text { (steradians) }\end{array}$} & \multirow[t]{2}{*}{$1-L($ not on disk $)$} & \multicolumn{2}{|c|}{$\begin{array}{c}\text { Eccentricity (All } \\
\text { Solutions) }\end{array}$} & \multicolumn{2}{|c|}{$\begin{array}{c}\text { Eccentricity (Disk } \\
\text { Solutions) }\end{array}$} & \multirow[t]{2}{*}{ Direction } \\
\hline & & & Peak & $3 \sigma$ Range & Peak & $3 \sigma$ Range & \\
\hline \multicolumn{8}{|c|}{ Candidate disk members } \\
\hline S2-16 & 0.47 & $7.89 \mathrm{e}-01$ & 0.60 & $0.00-1.00$ & 0.21 & $0.00-1.00$ & $\mathrm{CW}$ \\
\hline irs16SW-E & 0.18 & $6.76 \mathrm{e}-01$ & 0.37 & $0.00-1.00$ & 0.37 & $0.12-1.00$ & $\mathrm{CW}$ \\
\hline S1-14 & 0.12 & $5.80 \mathrm{e}-01$ & 0.33 & $0.00-1.00$ & 0.33 & $0.13-1.00$ & $\mathrm{CW}$ \\
\hline S2-6 & 0.15 & $5.76 \mathrm{e}-01$ & 0.79 & $0.17-1.00$ & 0.30 & $0.17-0.60$ & $\mathrm{CW}$ \\
\hline S3-5 & 0.20 & $5.62 \mathrm{e}-01$ & 0.64 & $0.00-1.00$ & 0.53 & $0.06-1.00$ & $\mathrm{CW}$ \\
\hline irs16SW & 0.14 & $5.38 \mathrm{e}-01$ & 0.78 & $0.04-1.00$ & 0.41 & $0.29-0.91$ & $\mathrm{CW}$ \\
\hline $\mathrm{S} 1-12$ & 0.10 & $4.56 \mathrm{e}-01$ & 0.41 & $0.00-1.00$ & 0.33 & $0.00-0.61$ & $\mathrm{CW}$ \\
\hline S2-4 & 0.13 & $4.23 \mathrm{e}-01$ & 0.69 & $0.10-1.00$ & 0.32 & $0.21-0.94$ & $\mathrm{CW}$ \\
\hline $\mathrm{S} 1-8$ & 0.09 & $4.06 \mathrm{e}-01$ & 0.62 & $0.37-1.00$ & 0.57 & $0.45-1.00$ & $\mathrm{CW}$ \\
\hline S1-2 & 0.10 & $3.66 \mathrm{e}-01$ & 0.26 & $0.00-1.00$ & 0.19 & $0.00-0.80$ & $\mathrm{CW}$ \\
\hline S2-17 & 0.21 & $3.63 \mathrm{e}-01$ & 0.77 & $0.00-1.00$ & 0.40 & $0.00-0.56$ & $\mathrm{CW}$ \\
\hline S3-10 & 0.08 & $3.21 \mathrm{e}-01$ & 0.16 & $0.00-1.00$ & 0.67 & $0.24-0.81$ & $\mathrm{CW}$ \\
\hline S2-7 & 0.54 & $3.02 \mathrm{e}-01$ & 0.76 & $0.00-1.00$ & 0.55 & $0.07-0.67$ & $\mathrm{CW}$ \\
\hline S3-25 & 0.50 & $2.64 \mathrm{e}-01$ & 0.76 & $0.00-1.00$ & 0.61 & $0.27-1.00$ & $\mathrm{CW}$ \\
\hline $\mathrm{S} 2-74$ & 0.24 & $2.01 \mathrm{e}-01$ & 0.45 & $0.00-1.00$ & 0.15 & $0.00-1.00$ & $\mathrm{CW}$ \\
\hline S1-21 & 0.14 & $1.67 \mathrm{e}-01$ & 0.92 & $0.00-1.00$ & 0.46 & $0.04-0.79$ & $\mathrm{CW}$ \\
\hline S2-19 & 0.22 & $1.60 \mathrm{e}-01$ & 0.57 & $0.00-1.00$ & 0.18 & $0.00-0.46$ & $\mathrm{CW}$ \\
\hline irs16CC & 0.17 & $1.49 \mathrm{e}-01$ & 0.62 & $0.34-1.00$ & 0.54 & $0.40-0.66$ & $\mathrm{CW}$ \\
\hline irs33E & 0.31 & $1.42 \mathrm{e}-01$ & 0.49 & $0.11-1.00$ & 0.50 & $0.27-1.00$ & $\mathrm{CW}$ \\
\hline $\mathrm{S} 1-3$ & 0.06 & $1.32 \mathrm{e}-01$ & 0.34 & $0.00-1.00$ & 0.09 & $0.00-1.00$ & $\mathrm{CW}$ \\
\hline S1-22 & 0.27 & $1.02 \mathrm{e}-01$ & 0.92 & $0.00-1.00$ & 0.68 & $0.34-0.83$ & $\mathrm{CW}$ \\
\hline S0-14 & 0.13 & $5.35 \mathrm{e}-02$ & $\ldots$ & $\ldots$ & $\ldots$ & $\ldots$ & $\mathrm{CW}$ \\
\hline \multicolumn{8}{|c|}{ Stars not in the disk } \\
\hline S0-15 & 0.12 & $1.55 \mathrm{e}-03$ & 0.30 & $0.00-1.00$ & $\ldots$ & $\ldots$ & $\mathrm{CW}$ \\
\hline $\operatorname{irs} 16 \mathrm{C}$ & 0.07 & $5.28 \mathrm{e}-04$ & 0.50 & $0.00-1.00$ & $\ldots$ & $\ldots$ & $\mathrm{CW}$ \\
\hline irs33N & 0.14 & $<1.00 \mathrm{e}-05$ & 0.97 & $0.00-1.00$ & $\ldots$ & $\ldots$ & $\mathrm{CW}$ \\
\hline $\mathrm{S} 1-24$ & 0.06 & $<1.00 \mathrm{e}-05$ & 0.98 & $0.13-1.00$ & $\ldots$ & $\ldots$ & $\mathrm{CCW}$ \\
\hline S3-19 & 0.57 & $<1.00 \mathrm{e}-05$ & 0.80 & $0.00-1.00$ & $\ldots$ & $\ldots$ & $\mathrm{CW}$ \\
\hline S3-30 & 0.03 & $<1.00 \mathrm{e}-05$ & 0.99 & $0.00-1.00$ & $\ldots$ & $\ldots$ & $\mathrm{CCW}$ \\
\hline irs 16NE & 0.14 & $<1.00 \mathrm{e}-05$ & 0.19 & $0.04-1.00$ & $\ldots$ & $\ldots$ & $\mathrm{CCW}$ \\
\hline irs16NW & 0.08 & $<1.00 \mathrm{e}-05$ & 0.70 & $0.00-1.00$ & $\ldots$ & $\ldots$ & $\mathrm{CCW}$ \\
\hline $\operatorname{irs} 29 \mathrm{~N}$ & 0.01 & $<1.00 \mathrm{e}-05$ & 0.99 & $0.00-1.00$ & $\ldots$ & $\ldots$ & $\mathrm{CCW}$ \\
\hline S2-66 & 0.53 & $<1.00 \mathrm{e}-05$ & 0.97 & $0.08-1.00$ & $\ldots$ & $\ldots$ & $\mathrm{CCW}$ \\
\hline
\end{tabular}

Note. ${ }^{\text {a }}$ No eccentricity is reported for S0-14 since the uniform- $a_{\rho}$ prior is not appropriate for this very low velocity star.

integrated over a region that has the same total area as the common plane,

$$
\begin{aligned}
L(\text { not on plane }) & =1-\frac{\int_{\text {plane }} \operatorname{PDF}(i, \Omega) d \mathrm{SA}}{\int_{\text {peak }} \operatorname{PDF}(i, \Omega) d \mathrm{SA}} \\
\int_{\text {plane }} d \mathrm{SA} & =\int_{\text {peak }} d \mathrm{SA},
\end{aligned}
$$

where SA is the solid angle and $L$ (not on plane) is the likelihood that the star is not on the common plane. Those stars with likelihoods, $L$ (not on plane), of greater than 0.9973 (equivalent to $3 \sigma$ for a Gaussian distribution) are flagged as nonmembers of the common plane. The remaining set of stars are considered candidate members of the common plane. Tables 3 and 4 list [1 $-L($ not on disk)] for each star and Figure 5 shows the positions of candidate members of the common plane in red and nonmembers in blue. Of the primary sample of 32 stars, 26 of which are orbiting in a clockwise sense on the plane-of-thesky, we find that 22 are possible members of the common plane $\left(N_{\text {disk-stars }}=22\right)$.

The clockwise common plane that we measure is slightly offset from the clockwise planes proposed in earlier works.
Overplotted in black in Figure 12 is the candidate orbital plane proposed by Levin \& Beloborodov (2003) with updated values from Paumard et al. (2006) for the candidate plane normal vector (solid black) and thickness (dashed black). The previously proposed plane was derived by minimizing a statistical metric, $K$, in order to find the best-fit common orbital plane from the velocity vectors of a sample of stars (see Appendix C). However, some stars are not members of the common plane and including them in the fit biases the result since they have extremely wellmeasured velocities (S0-15, IRS 16C, S3-19). For example, using the $K$ metric approach of Levin \& Beloborodov (2003), fitting all 26 clockwise stars in our primary sample gives $i=128^{\circ}$ and $\Omega=102^{\circ}$ with $K=0.7$, which is closer to the disk found by Paumard et al. (2006) at $i=127^{\circ}$ and $\Omega=99^{\circ}$. While fitting only the 22 stars that are consistent with the clockwise disk based on our orbit analysis gives $i=117^{\circ}$ and $\Omega=98^{\circ}$ with $K=0.2$. Therefore, using the $K$ metric to determine the common plane can produce biased results due to the inclusion of nonmembers. By combining position, velocity, and acceleration information in order to determine the orbital plane for each star, the direction of a common orbital plane can be estimated more robustly. 
Table 4

Orbital Eccentricity and Disk Membership for Stars Added to the Extended Sample

\begin{tabular}{|c|c|c|c|c|c|c|c|}
\hline \multirow[t]{2}{*}{ Name } & \multirow{2}{*}{$\begin{array}{c}\mathrm{SA}_{\vec{n}} \\
\text { (steradians) }\end{array}$} & \multirow[t]{2}{*}{$1-L($ not on disk) } & \multicolumn{2}{|c|}{$\begin{array}{l}\text { Eccentricity (All } \\
\text { Solutions) }\end{array}$} & \multicolumn{2}{|c|}{$\begin{array}{c}\text { Eccentricity (Disk } \\
\text { Solutions) }\end{array}$} & \multirow[t]{2}{*}{ Direction } \\
\hline & & & Peak & $3 \sigma$ Range & Peak & $3 \sigma$ Range & \\
\hline \multicolumn{8}{|c|}{ Candidate disk members } \\
\hline paumE57 & 0.41 & $8.57 \mathrm{e}-01$ & 0.29 & $0.00-1.00$ & 0.34 & $0.00-0.91$ & $\mathrm{CW}$ \\
\hline irs34W & 0.21 & $5.12 \mathrm{e}-01$ & 0.20 & $0.00-1.00$ & 0.20 & $0.00-1.00$ & $\mathrm{CW}$ \\
\hline paumE72 & 1.50 & $4.31 \mathrm{e}-01$ & 0.81 & $0.00-1.00$ & 0.56 & $0.00-1.00$ & $\mathrm{CW}$ \\
\hline paumE73 & 0.98 & $2.41 \mathrm{e}-01$ & 0.02 & $0.00-1.00$ & 0.96 & $0.00-1.00$ & $\mathrm{CW}$ \\
\hline irs34NW & 0.39 & $2.33 \mathrm{e}-01$ & 0.04 & $0.00-1.00$ & 0.07 & $0.00-1.00$ & $\mathrm{CW}$ \\
\hline AFNWNW & 1.52 & $2.06 \mathrm{e}-01$ & 0.99 & $0.00-1.00$ & 0.96 & $0.00-1.00$ & CW \\
\hline paumE69 & 0.51 & $1.34 \mathrm{e}-01$ & 0.02 & $0.00-1.00$ & 0.80 & $0.12-1.00$ & $\mathrm{CW}$ \\
\hline irs9SW & 0.60 & $1.10 \mathrm{e}-01$ & 0.05 & $0.00-1.00$ & 0.35 & $0.00-1.00$ & $\mathrm{CW}$ \\
\hline paumE54 & 0.36 & $1.03 \mathrm{e}-01$ & 0.08 & $0.00-1.00$ & 0.17 & $0.00-0.68$ & $\mathrm{CW}$ \\
\hline $\operatorname{irs} 1 \mathrm{E}$ & 0.88 & $7.35 \mathrm{e}-02$ & 0.77 & $0.00-1.00$ & 0.93 & $0.56-1.00$ & $\mathrm{CW}$ \\
\hline irs9W & 0.57 & $6.29 \mathrm{e}-02$ & 0.02 & $0.00-1.00$ & 0.67 & $0.17-1.00$ & $\mathrm{CW}$ \\
\hline paumE87 & 0.65 & $5.09 \mathrm{e}-02$ & 0.09 & $0.00-1.00$ & 0.94 & $0.52-1.00$ & $\mathrm{CW}$ \\
\hline irs15SW & 0.35 & $2.64 \mathrm{e}-02$ & 0.02 & $0.00-1.00$ & 0.94 & $0.31-1.00$ & $\mathrm{CW}$ \\
\hline $\mathrm{AF}$ & 0.23 & $1.22 \mathrm{e}-02$ & 0.11 & $0.00-1.00$ & 0.99 & $0.77-1.00$ & $\mathrm{CCW}$ \\
\hline irs $1 \mathrm{~W}$ & 0.59 & $9.61 \mathrm{e}-03$ & 0.18 & $0.00-1.00$ & 0.87 & $0.35-1.00$ & $\mathrm{CW}$ \\
\hline irs7SW & 0.06 & $8.14 \mathrm{e}-03$ & 0.99 & $0.00-1.00$ & 0.95 & $0.68-1.00$ & $\mathrm{CW}$ \\
\hline S3-26 & 0.22 & $3.32 \mathrm{e}-03$ & 1.00 & $0.00-1.00$ & 0.92 & $0.74-1.00$ & $\mathrm{CW}$ \\
\hline \multicolumn{8}{|c|}{ Stars not in the disk } \\
\hline AFNW & 1.08 & $1.31 \mathrm{e}-03$ & 0.95 & $0.00-1.00$ & $\ldots$ & $\ldots$ & $\mathrm{CW}$ \\
\hline irs 15NE & 1.07 & $8.92 \mathrm{e}-04$ & 0.99 & $0.00-1.00$ & $\ldots$ & $\ldots$ & $\mathrm{CCW}$ \\
\hline paumE78 & 0.61 & $4.06 \mathrm{e}-04$ & 0.98 & $0.00-1.00$ & $\ldots$ & $\ldots$ & $\mathrm{CCW}$ \\
\hline irs9SE & 0.89 & $7.12 \mathrm{e}-05$ & 0.02 & $0.00-1.00$ & $\ldots$ & $\ldots$ & $\mathrm{CCW}$ \\
\hline irs7E2? & 0.56 & $6.80 \mathrm{e}-05$ & 0.99 & $0.00-1.00$ & $\ldots$ & $\ldots$ & $\mathrm{CW}$ \\
\hline paumE84 & 0.22 & $4.09 \mathrm{e}-05$ & 0.98 & $0.00-1.00$ & $\ldots$ & $\ldots$ & $\mathrm{CW}$ \\
\hline paumE89 & 0.94 & $<1.00 \mathrm{e}-05$ & 0.02 & $0.00-1.00$ & $\ldots$ & $\ldots$ & $\mathrm{CCW}$ \\
\hline irs13E1 & 0.50 & $<1.00 \mathrm{e}-05$ & 0.98 & $0.00-1.00$ & $\ldots$ & $\ldots$ & $\mathrm{CCW}$ \\
\hline paumE86 & 0.32 & $<1.00 \mathrm{e}-05$ & 0.06 & $0.00-1.00$ & $\ldots$ & $\ldots$ & $\mathrm{CCW}$ \\
\hline paumE82 & 0.79 & $<1.00 \mathrm{e}-05$ & 0.02 & $0.00-1.00$ & $\ldots$ & $\ldots$ & $\mathrm{CCW}$ \\
\hline paumE75 & 0.42 & $<1.00 \mathrm{e}-05$ & 0.99 & $0.00-1.00$ & $\ldots$ & $\ldots$ & $\mathrm{CW}$ \\
\hline paumE64 & 0.67 & $<1.00 \mathrm{e}-05$ & 0.62 & $0.00-1.00$ & $\ldots$ & $\ldots$ & $\mathrm{CW}$ \\
\hline paumE62 & 0.58 & $<1.00 \mathrm{e}-05$ & 0.02 & $0.00-1.00$ & $\ldots$ & $\ldots$ & $\mathrm{CCW}$ \\
\hline paumE60 & 0.21 & $<1.00 \mathrm{e}-05$ & 0.99 & $0.00-1.00$ & $\ldots$ & $\ldots$ & $\mathrm{CCW}$ \\
\hline paumE55 & 0.61 & $<1.00 \mathrm{e}-05$ & 0.95 & $0.00-1.00$ & $\ldots$ & $\ldots$ & $\mathrm{CCW}$ \\
\hline paumE53 & 0.49 & $<1.00 \mathrm{e}-05$ & 0.97 & $0.00-1.00$ & $\ldots$ & $\ldots$ & $\mathrm{CW}$ \\
\hline paumE52 & 0.26 & $<1.00 \mathrm{e}-05$ & 0.09 & $0.00-1.00$ & $\ldots$ & $\ldots$ & $\mathrm{CCW}$ \\
\hline paumE42 & 0.59 & $<1.00 \mathrm{e}-05$ & 0.04 & $0.00-1.00$ & $\ldots$ & $\ldots$ & $\mathrm{CCW}$ \\
\hline irs $7 \mathrm{~W}$ & 0.33 & $<1.00 \mathrm{e}-05$ & 0.98 & $0.00-1.00$ & $\ldots$ & $\cdots$ & $\mathrm{CCW}$ \\
\hline irs7SE & 0.81 & $<1.00 \mathrm{e}-05$ & 0.07 & $0.00-1.00$ & $\ldots$ & $\ldots$ & $\mathrm{CCW}$ \\
\hline irs7E1(ESE) & 1.18 & $<1.00 \mathrm{e}-05$ & 0.05 & $0.00-1.00$ & $\ldots$ & $\ldots$ & $\mathrm{CCW}$ \\
\hline irs13E4 & 0.60 & $<1.00 \mathrm{e}-05$ & 0.70 & $0.00-1.00$ & $\ldots$ & $\ldots$ & $\mathrm{CCW}$ \\
\hline $\operatorname{irs} 13 \mathrm{E} 3 \mathrm{~b}$ & 0.53 & $<1.00 \mathrm{e}-05$ & 0.64 & $0.00-1.00$ & $\ldots$ & $\ldots$ & $\mathrm{CCW}$ \\
\hline irs13E2 & 0.59 & $<1.00 \mathrm{e}-05$ & 0.60 & $0.00-1.00$ & $\ldots$ & $\ldots$ & $\mathrm{CCW}$ \\
\hline
\end{tabular}

The detected common orbital plane is composed of stars dispersed in a disk rather than in a single cluster as can be seen from the stars' positions within the common plane shown in Figure 13. In this figure, the stars' positions have been converted into a disk coordinate system defined as $[\hat{p}, \hat{q}, \hat{n}]$ where $\hat{n}$ is perpendicular to the disk plane, $\hat{p}$ is along the line of ascending nodes (where the plane of the sky intersects the disk plane), and $\hat{q}=\hat{n} \times \hat{p}$. For each star, all orbital solutions that fall within $10^{\circ}$ of the common orbital plane are combined to create a probability distribution for the star's position in the disk, $\operatorname{PDF}(p, q)$, which is shown in Figure 13 (left). Each stars probability distribution is elongated in the $q$-direction due to the large range of line-of-sight distances, $z$, that are possible within the small range of possible disk inclinations for this nearly edge-on plane of the disk. The thickness in the $p$ direction is largely set by the uncertainties in the potential parameters $\left(M_{\bullet}, R_{o}\right)$ and velocities. The distribution of young stars within the plane shows a range of position angles on the plane, consistent with a stellar disk rather than a stellar cluster.

The CW stellar disk is detected both in our analysis of the primary sample and in a similar analysis of the entire extended sample. The additional young stars in the extended sample have larger velocity uncertainties and no acceleration information. Therefore, the Monte Carlo orbit analysis samples from a prior probability distribution that is uniform in acceleration, ranging from the largest allowed by the projected radius to the smallest allowed for the orbit to remain bound. We note that even if we ignore the acceleration measurements for our primary sample analysis, the CW stellar disk is still detected, although the significance is lowered from $\sim 19 \sigma$ to $\sim 8 \sigma$ above the background density of normal vectors. Thus the additional stars' orbits in the extended sample are still constrained (see Figure 14), even though they have larger uncertainties as compared to the 


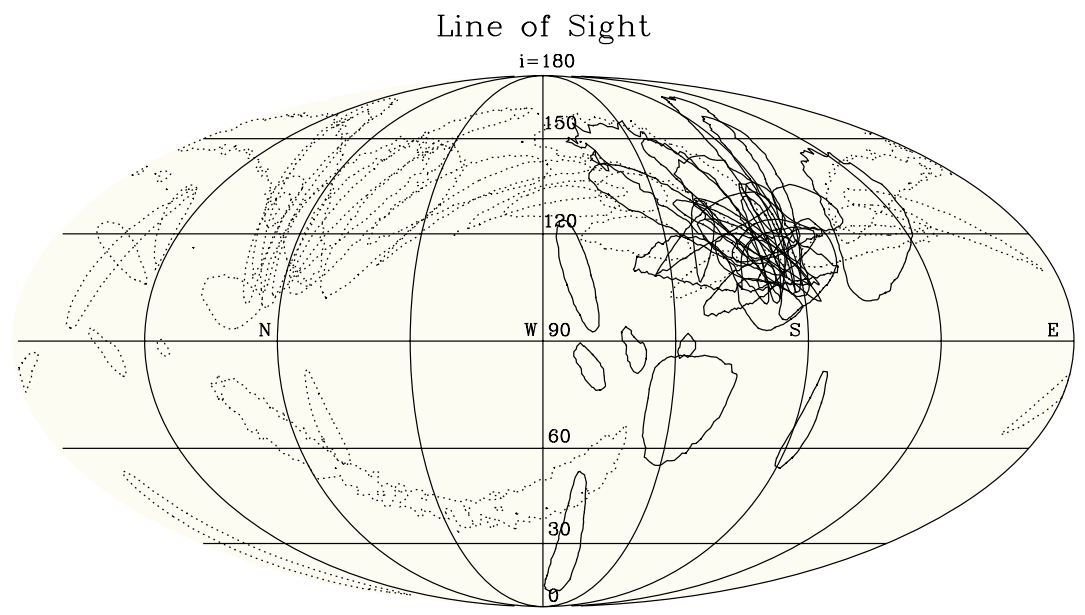

To Sun

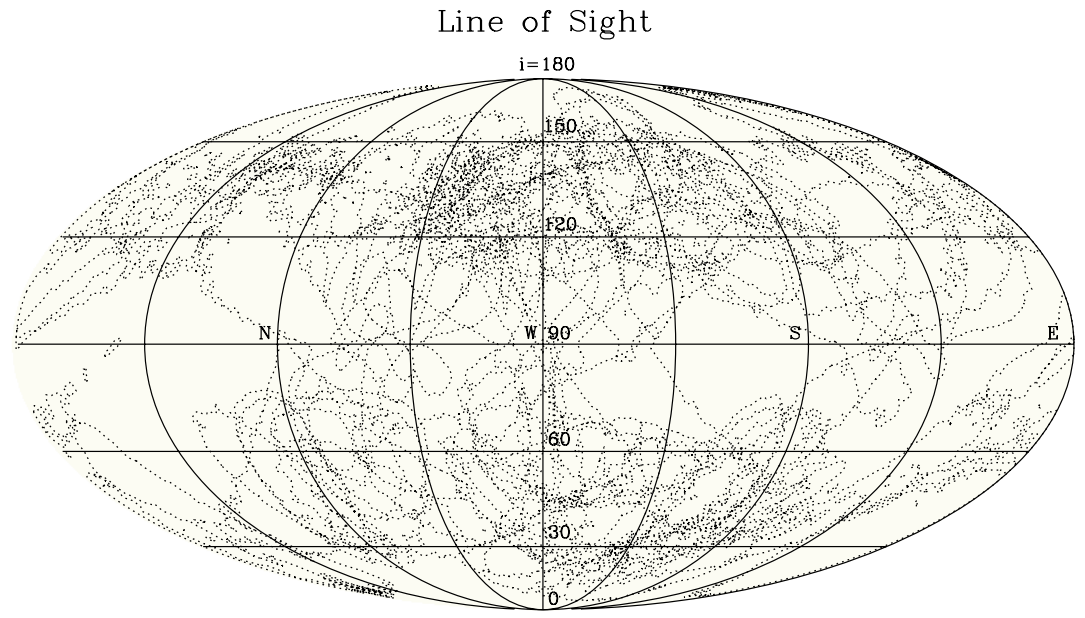

To Sun

Figure 11. $1 \sigma$ contours of all stars' probability distribution functions for the orientation of their orbital planes. This shows the distribution of stellar orbit orientations around the sky. The primary sample is plotted on the top and if there are degenerate solutions for a given star, then one solution is plotted with a solid line and the other with a dashed line. Additional sources found only in the secondary sample are plotted on the bottom and are plotted with dashed lines as there are no acceleration constraints and each star has a single solution with large uncertainties. We note that the orientation of the projection shown in this figure is rotated by $180^{\circ}$ with respect to that shown in earlier publications (e.g., Eisenhauer et al. 2005; Nayakshin et al. 2006) in order to more easily see the region around the proposed disks.

(A color version of this figure is available in the online journal.)

stars in just the primary sample. The density of normal vectors from the extended sample analysis shows a peak within $1^{\circ}$ of the disk's position from the primary sample.

The analysis of the extended sample shows that $\sim 50 \%$ of the young stars reside on the $\mathrm{CW}$ disk and there is no statistically significant change $(>3 \sigma)$ in the fractional number of disk stars at different radii. For reference, the 73 young stars in the extended sample are distributed on the plane of the sky with a surface density that decreases with radius as $\rho^{-2.1 \pm 0.4}$. Within a projected radius of $3^{\prime \prime}$, the fraction of candidate disk members is $72 \% \pm 9 \%$ (18 out of 25) and at projected radii larger than $3^{\prime \prime}$, the fraction of candidate disk members is $42 \% \pm 7 \%$ (20 out of 48). Given the small number of known young stars, Poisson statistics indicate that this change in the fraction of candidate disk members is only marginally statistically significant at the $2.6 \sigma$ level. Likewise, the projected surface density for the ondisk and off-disk populations shown no significant difference from each other or from that of the total population. Thus, the number of candidate disk members does not change with radius and roughly half of the young stars reside on the $\mathrm{CW}$ disk.

The KLF of the young stars does not change significantly with radius or when considering stars on and off the disk. To compare the KLF as a function of radius, the entire extended sample of young stars is divided into a near sample $(r<3.5)$ and a far sample $(r \geqslant 3$ '.5) and the KLF is constructed for each. A twosample KS test yields a probability of $46 \%$ that the near and far samples have the same KLF. Similarly, the KLF is constructed for stars on and off the disk and a two-sample KS test yields a probability of $74 \%$ that the on-disk and off-disk samples have the same KLF. Finding more young stars will allow for a more detailed comparison of the KLF for different subsets within the young stars population.

\subsection{Limits on Additional Stellar Disks}

In our primary sample, no common orbital plane is detected for the counterclockwise population of stars; however, our sample is limited to six counterclockwise orbiting stars, only two of which (IRS 16NE, IRS 16NW) are claimed by Paumard et al. (2006) to reside on the counterclockwise disk. Out of the six counterclockwise stars in our primary sample, we find that only IRS 16NE and S2-66 could be consistent with the previously proposed counterclockwise disk. The proposed counterclockwise disk may have a larger radial extent than is covered by our observations, so in order to fully explore whether 

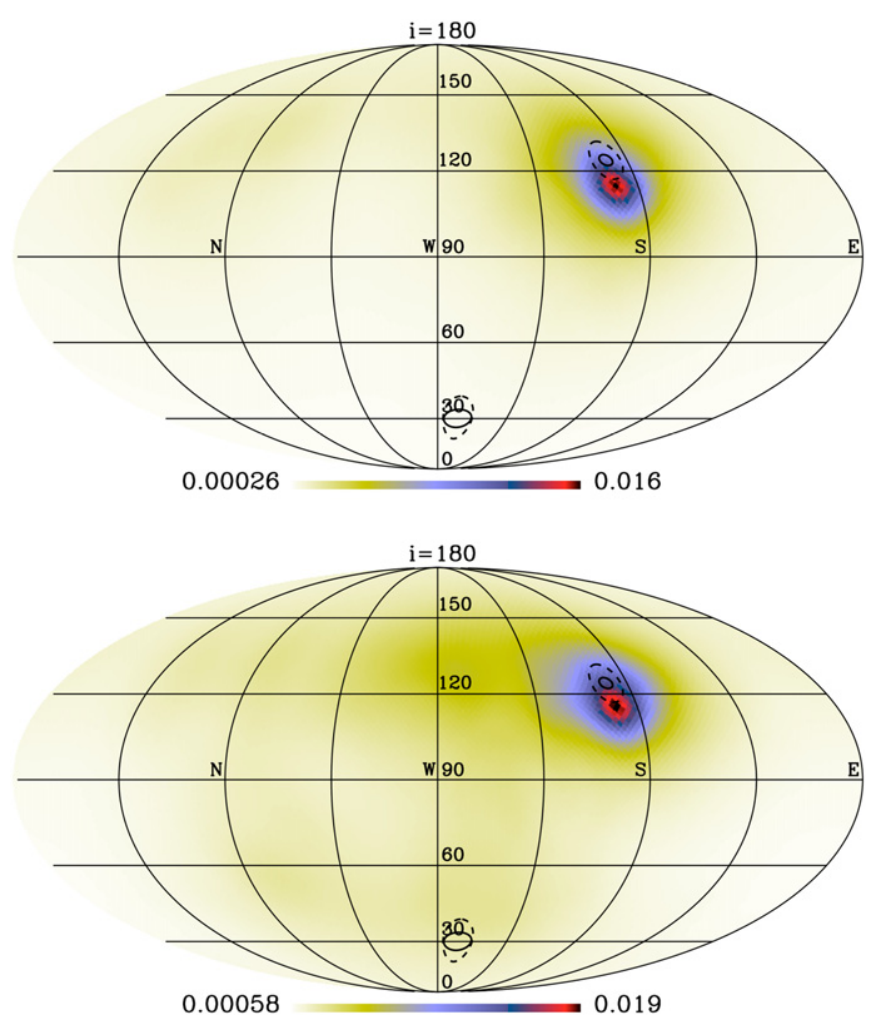

Figure 12. Density of normal vectors to the orbital planes of the stars in our primary (top) and extended (bottom) samples. Densities are indicated in colors (stars $\mathrm{deg}^{-2}$ ) on a linear scale and the peak indicates an overdensity of stars with similar orbital planes. Overplotted in black are the candidate orbital planes as proposed by Levin \& Beloborodov (2003) and Genzel et al. (2003) with updated values from Paumard et al. (2006) for the candidate plane normal vector and uncertainties (solid black) and the disk thickness (dashed black) shown as solid angles of $0.05 \mathrm{sr}$ and $0.09 \mathrm{sr}$ for the clockwise and counterclockwise disks, respectively.

our lack of detection of a second disk is due to our limited field of view, it is necessary to analyze the extended sample. As discussed in Section 4, the uniform acceleration prior adopted for this analysis tends to overemphasize face-on orbital planes, making it easier to detect the proposed CCW disk, as Paumard et al. (2006) suggest it has an inclination of $24^{\circ}$.

Using the extended sample, our analysis of the density of normal vectors, in the region of the proposed counterclockwise disk, reveals no significant overdensity. Of the 73 stars in the extended sample, at least 34 are not on the clockwise disk and thus we compare the density observed in the region of the proposed counterclockwise disk to that expected for an isotropic distribution of 34 stars. The observed density of normal vectors in the region of the counterclockwise disk is $2.4 \times 10^{-3} \mathrm{stars} \mathrm{deg}^{-2}$, which is only a factor of 3 above what is expected for an isotropic distribution and is less than $1 \sigma$ above the background over the rest of the sky (excluding the clockwise peak). This density of normal vectors corresponds to only three stars within $19^{\circ}$ of the putative CCW disk, where $19^{\circ}$ is the disk thickness proposed by Paumard et al. (2006), and is consistent with random fluctuations of an isotropic distribution having the $\vec{n}$-uncertainties shown in Figure 14. We estimate that this analysis is capable of revealing, at the $3 \sigma$ level, a stellar disk with more than seven stars within a solid angle cone of radius $=19^{\circ}$ at the location of the proposed CCW disk; thus the proposed CCW disk containing 17 stars as suggested by Paumard et al. (2006) should have been detected with this approach.

There are several principal differences between our analysis and that in earlier works. First, previous works make the a priori assumption that a disk exists through the use of the statistical metric, $K$, and the results were not compared to a null hypothesis (i.e., no disk) to establish the statistical significance of a disk detection. Furthermore, the $K$ metric used in previous works suffers from a bias which is described in Appendix C. The primary goal of our methodology is to minimize the number of a priori assumptions and to fully quantify the significance of any disk detected as compared to the null hypothesis that there is no disk. Therefore, we choose to search for disks using all the young stars rather than first trimming out stars based on projected angular momentum criteria or radii. Also, we determine the range of allowed orbital orientations for each star individually
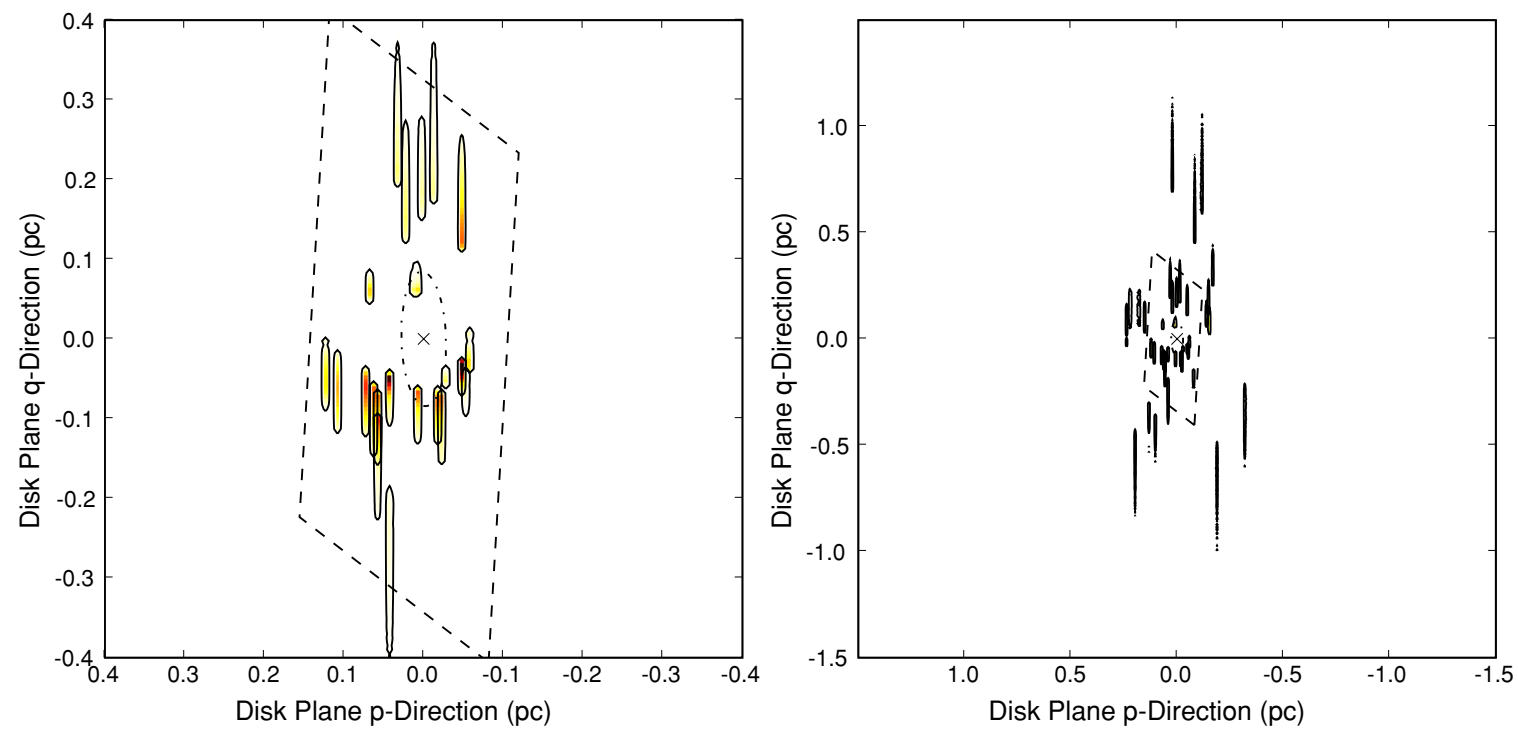

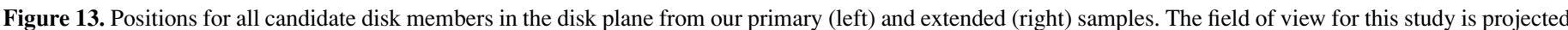

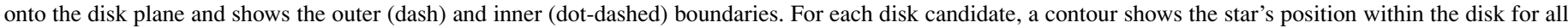

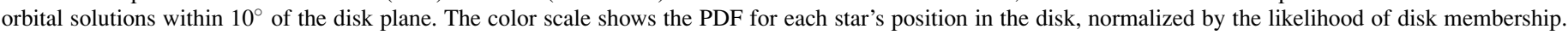
This normalization shows stars with a higher and lower likelihood of disk membership as darker red or lighter yellow, respectively.

(A color version of this figure is available in the online journal.) 


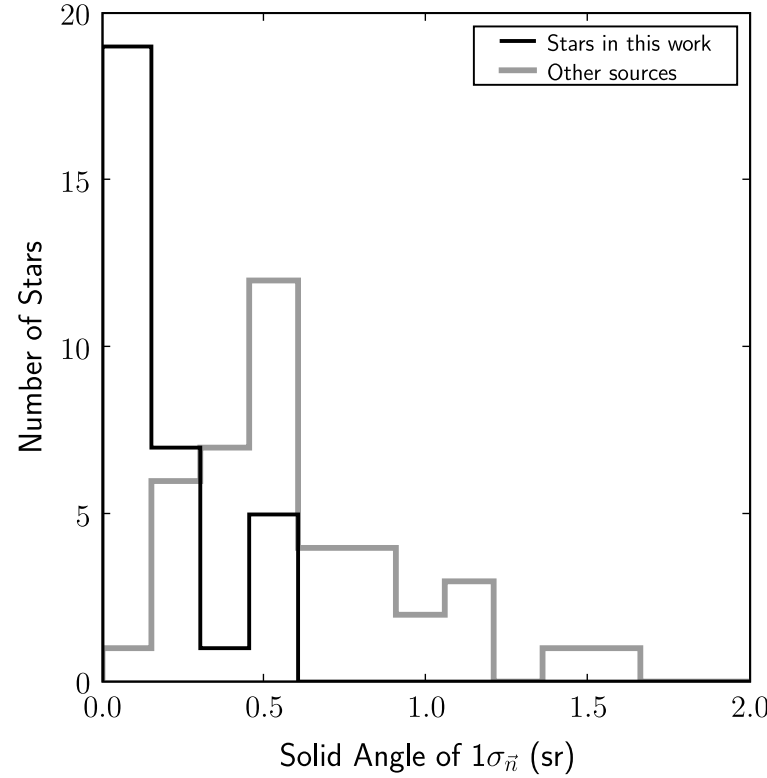

Figure 14. Distribution of $\vec{n}$-uncertainties as expressed by the area of the $1 \sigma$ region in which a star's normal vector can point. The uncertainties are shown both for the sample in this work (black) and for the stars with only threedimensional position and two-dimensional velocity information extracted from Paumard et al. (2006) that are used in the search for a second disk (gray)

rather than searching for a disk from a statistical sample of young stars. In this fashion, we utilize not only the direction information for a velocity vector, as has been used previously, but also the physical relationships between the magnitude of the velocity and the positional information. This method allows for no disk to be detected, while the previously used statistical tests assumed a disk model and, therefore, must be compared to the no-disk hypothesis using simulations of isotropic populations. Without the simulations, the significance of any disk detection via the $K$ metric cannot be fully quantified. The resulting distribution of orbits from our analysis is consistent with the hypothesis of a single, clockwise disk plus a more randomly distributed population.

\subsection{Properties of the Clockwise Disk}

We now examine, in detail, the properties of the detected clockwise disk. With the identification of a single stellar disk and a candidate list of disk members, we investigate the following: (1) the thickness of the disk, (2) the radial profile of the disk, (3) the azimuthal isotropy of the disk, (4) the eccentricities of stars in the disk, and (5) the luminosity function of the stars in the disk. These properties are critical for distinguishing between in situ and infalling cluster formation scenarios, as well as for understanding the dynamical evolution of the young stars both on and off the disk.

The observed disk of young stars has a significant intrinsic thickness; however, the vertical velocity dispersion is less than previously determined. To measure the thickness of the disk, the dispersion of the velocities out of the plane (along the $\vec{n}$ direction) is calculated from all candidate disk members by projecting each star's three-dimensional velocity vector along the disk's normal vector to give $v_{\vec{n}}$. The measurement uncertainties in both $\vec{v}$ and $\vec{n}$ are propagated through this coordinate transformation. The intrinsic velocity dispersion is calculated using

$$
\sigma_{\vec{n}, \text { intrinsic }}^{2}=\sigma_{\vec{n}, \text { measured }}^{2}-\sigma_{\vec{n}, \text { bias }}^{2}
$$

$$
\begin{aligned}
\sigma_{\vec{n}, \text { intrinsic }}^{2}= & \left(\frac{1}{N_{\text {disk-stars }}-1}\right) \\
& \times\left(\sum_{i=0}^{N_{\text {disk-stars }}} v_{\vec{n}, i}^{2}-\sum_{i=0}^{N_{\text {disk-stars }}} \operatorname{error}^{2}\left(v_{\vec{n}, i}\right)\right),
\end{aligned}
$$

where the bias term, $\sigma_{\vec{v}}$,bias, is $19 \mathrm{~km} \mathrm{~s}^{-1}$ and accounts for added dispersion as a result of uncertainties in the measurements. The resulting intrinsic velocity dispersion is $28 \pm 6 \mathrm{~km} \mathrm{~s}^{-1}$, which is significantly different from zero, thus a finite thickness is required. However, this velocity dispersion is a factor of 2 smaller than that found using the previously proposed disk solution of Paumard et al. (2006) and is slightly smaller than the value reported in Beloborodov et al. (2006) due to our improved identification of candidate disk members. The disk's thickness can be expressed as the ratio of the vertical scale height to radius, $h / r=\sigma_{\vec{n}}$,intrinsic $/\langle|\vec{v}|\rangle$, and is $0.08 \pm 0.02$. Following a similar analysis to Beloborodov et al. (2006), but with the above relationship between $h / r$ and the velocity dispersion, the disk thickness can also be described using a Gaussian distribution of inclination angles about the disk plane with a standard deviation of $\Delta \theta$ and is related to the scale height of the disk by $h / r \sim \sqrt{1 / 2} \Delta \theta$. This yields a dispersion angle of $\Delta \theta=7^{\circ} \pm 2^{\circ}$ for the young stellar disk. This more rigorous determination of the disk thickness is consistent with the thickness we derived in Section 5.1 from the half-width at half-maximum of the peak in the density of normal vectors; thus the selection of the candidate disk members is likely robust. In comparison, the previously proposed disk solutions yield a disk thickness of $h / r=0.2$ $\left(\Delta \theta=14^{\circ}\right)$ and $h / r=0.1\left(\Delta \theta=9^{\circ}\right)$ for Paumard et al. (2006) and Beloborodov et al. (2006), respectively. We caution that all of these conversions from velocity dispersion to disk scale height and dispersion angle assume circular orbits and an isothermal disk structure. From our analysis, we note that the out-of-the-plane velocity dispersion shows no statistically significant variation with radius in the disk both for the primary (difference is $1 \sigma \sim 7 \mathrm{~km} \mathrm{~s}^{-1}$ ) and the full extended samples (difference is $1 \sigma \sim 14 \mathrm{~km} \mathrm{~s}^{-1}$ ). Therefore, the observations are consistent with a thin disk of uniform velocity dispersion at all radii.

The surface density of stars in the disk falls off rapidly as a function of radius. In order to extend the radial coverage, we consider the entire extended sample in this analysis. The young stars that are candidate disk members have constraints on their three-dimensional radii if we limit their orbital solutions to those close to the disk plane. Thus, the disk's surface density can be determined as a function of three-dimensional radius rather than just the projected two-dimensional radius as discussed at the end of Section 5.1. The distribution for each star's position within the disk plane, $\operatorname{PDF}(p, q)$, is constructed from orbits that are within $10^{\circ}$ of the disk and is shown in Figure 13. Then the disk's surface density at each radius is computed numerically by sampling the $\operatorname{PDF}(p, q) 10^{5}$ times for all the candidate disk members and constructing a radial histogram for each trial. The radial histograms are combined for all the trials to find the peak and $68 \%$ confidence bounds for the expected number of stars at each radius. This is converted into an azimuthally integrated surface density by dividing by the area of a ring at each radius. This method of constructing the surface density captures both the measurement error in the individual stars and the finite thickness of the disk, which has not been incorporated into previous estimates. The resulting azimuthally averaged surface density on the disk is shown for the extended sample in Figure 15 


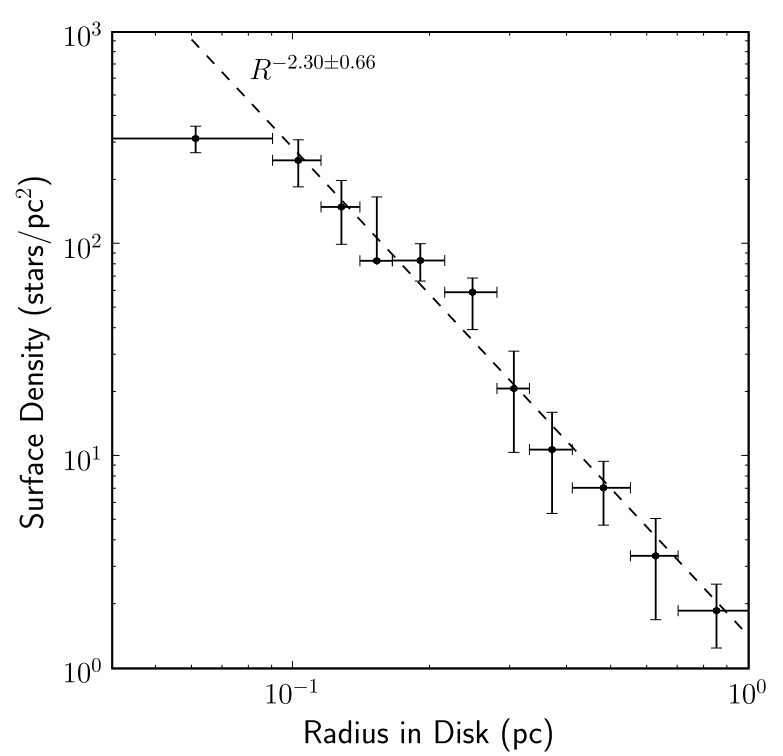

Figure 15. Radial distribution of stars within the disk plane for the extended sample. The best-fit line is shown (dashed) and was constructed by excluding the first data point and the last three data points where field of view limitations may affect the distribution.

and has a best-fit power-law profile of $r^{-2.3 \pm 0.7}$. This is consistent with the previous results (Paumard et al. 2006), but our analysis includes the uncertainty in each stars line-of-sight distance due to the finite disk thickness and, therefore, yields a larger uncertainty on the power-law index.

Visual examination of the stars' positions in the disk plane (Figure 13) suggests there may be some anisotropy as evidenced by the clustering of stars on the lower part of the disk; however, this overdensity is only marginally statistically significant based on the following analysis. In order to search for nonuniformities, we compare the observed stellar surface density of the extended sample within the disk plane with the surface density expected for an azimuthally symmetric disk. The observed stellar surface density is measured by sampling from all stars' $\operatorname{PDF}(p, q)$ for $10^{5}$ trials and calculating the stellar surface density over a grid of points in the disk plane for each trial. For each point on the disk plane, the surface densities from all trials are combined, yielding the most probable surface density with uncertainties. The resulting two-dimensional map of observed surface densities is then compared to the expected surface densities for an azimuthally symmetric disk by subtracting the two values and dividing by the uncertainties. This produces a surface density excess map that shows the significance of any excess. The disk shows a marginally significant $(\sim 3 \sigma)$ overdensity on the front side $(q<0)$ of the disk and a corresponding underdensity on the back side $(q>0)$.

A few candidate disk stars show evidence for eccentric orbits. To determine whether any of the stars' eccentricities are consistent with a circular orbit, the six-dimensional PDF for the orbital parameters is marginalized and re-expressed as a PDF for the eccentricity vector (see Appendix B), PDF $\left(e_{x}, e_{y}, e_{z}\right)$. The magnitude of this vector is the orbital eccentricity and the direction points along the semimajor axis toward the periapse position. The PDF for the eccentricity vector cannot be further marginalized to produce a PDF of the eccentricity magnitude without introducing a bias due to the positive, definite nature of a vector magnitude. This is the same bias term as described in the velocity dispersion analysis; however, unlike the velocities, the eccentricity distributions are strongly
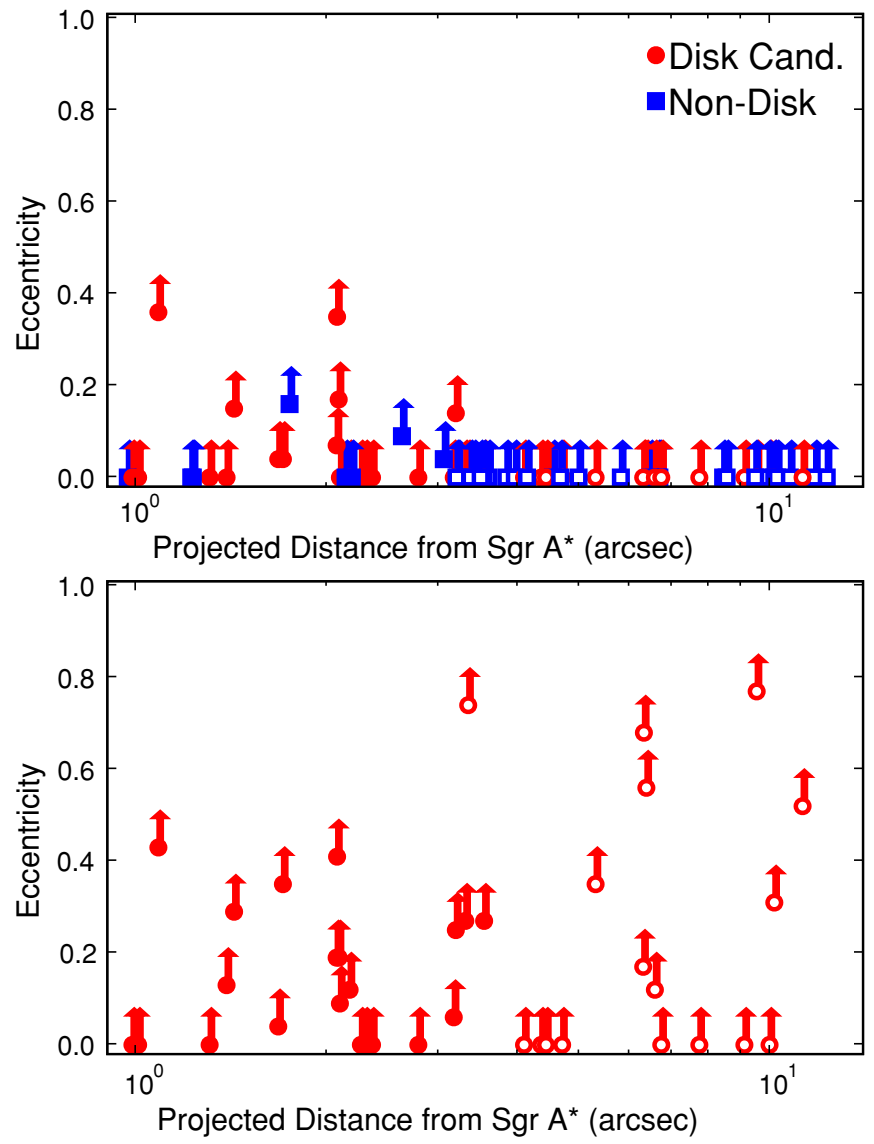

Figure 16. Distribution of eccentricity lower limits as determined from individual stellar orbits, excluding S0-14. The top panel shows the $99.7 \%$ confidence lower limit from all possible orbital solutions for candidate disk members (red circles) and nondisk members (blue squares). Stars from the primary sample (filled) and stars added in the extended sample (unfilled) are both shown. Sources in only the extended sample have less-constrained eccentricities due to their larger velocity uncertainties. The bottom panel shows the candidate disk members $99.7 \%$ confidence lower limits after restricting the orbital solutions to those with normal vectors within $10^{\circ}$ of the disk. By assuming disk membership, the range of eccentricities is more restricted for the candidate disk members.

(A color version of this figure is available in the online journal.)

non-Gaussian and the bias term cannot easily be accounted for in the marginalization. The peak of $\operatorname{PDF}\left(e_{x}, e_{y}, e_{z}\right)$ gives the unbiased orbital eccentricity and the $99.7 \%$ confidence interval of the three-dimensional distribution is used to determine the range for the one-dimensional eccentricity. Tables 3 and 4 show the $99.7 \%$ confidence range of the eccentricities for all stars in the primary and extended samples. Also, Figure 16 shows the eccentricity $99.7 \%$ confidence lower limit for the candidate disk members in red, nondisk members in blue, and excludes S0-14 (see Section 4). When considering all possible orbital solutions, the resulting eccentricity ranges show that two candidate disk members from the primary sample have $99.7 \%$ confidence eccentricity lower limits of greater than 0.2 . Restricting the possible orbital solutions to only those having normal vectors oriented within $10^{\circ}$ of the disk normal vector increases the number to eight candidate disk members with 99.7\% confidence eccentricity lower limits larger than 0.2 .

We find high-eccentricity stars in the disk, similar to the analysis of Beloborodov et al. (2006) in which they assumed an infinitely thin disk. However, our analysis incorporates the finite thickness of the disk and places statistical errors on the eccentricities for individual stars. 


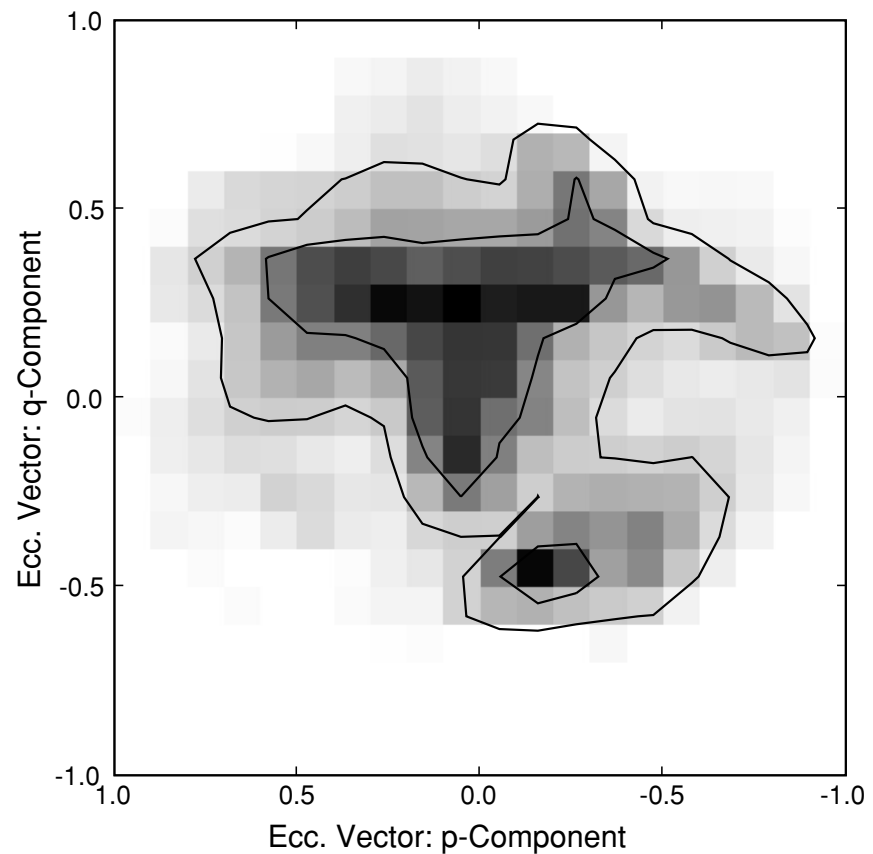

Figure 17. Combined probability distribution for the candidate disk stars' eccentricity vectors. The eccentricity vectors for orbital solutions with normal vectors within $10^{\circ}$ of the disk's normal vector are projected onto the disk plane. The $1 \sigma$ and $2 \sigma$ confidence-level contours are shown in black.

The average eccentricity of the entire population is not yet well constrained. The eccentricity for the stellar disk is determined using the eccentricity vector. For each candidate disk member, orbital solutions are selected whose normal vectors point within $10^{\circ}$ of the disk normal vector. These orbital solutions are combined for all the disk stars by averaging their PDFs to create a combined probability distribution for all stars' eccentricity vectors, which is then projected into the disk plane and plotted in two dimensions (Figure 17). This two-dimensional probability distribution gives an unbiased estimate of the eccentricity magnitude and shows that while the characteristic disk eccentricity peaks at $e=0.22$, it is consistent with $e=0.0-0.8$ at the $1 \sigma$ level, reflecting the large eccentricity uncertainties for the majority of the candidate disk members.

\section{DISCUSSION}

The kinematic analysis of the young stars in the central parsec around our Galaxy's supermassive black hole has implications for the recent star-formation history in this region. Our first attempt at determining individual orbits for young stars that reside outside the central arcsecond shows definitive evidence for the clockwise-rotating disk that was suggested by Levin \& Beloborodov (2003) and was subsequently refined by Genzel et al. (2003) and Paumard et al. (2006). Our results do not show a statistically significant second disk. The presence of a single stellar disk eliminates the need to invoke two distinct starburst events occurring roughly $6 \mathrm{Myr}$ ago and greatly simplifies the demands on both in situ and infalling cluster scenarios. For instance, in the self-gravitating gas disk scenario, the detection of only a single stellar disk lifts the requirement for a second disk to rapidly build up gas, fragment, and form stars within 1-2 Myr of the formation of the first disk. Likewise, for the infalling cluster scenario, the presence of only one stellar disk means that the frequency of such infall events is half that required for the existence of two disks. On the strength of our confirming only one stellar disk, we consider whether all of the young stars within the central parsec may have formed in a single burst of star formation.

Such a scenario must explain not only the observed clockwise stellar disk, oriented at $i \sim 115^{\circ}$ and $\Omega \sim 100^{\circ}$, but also the presence of roughly half of the young stars from our extended sample on more isotropically distributed orbits out of the disk. In the single starburst scenario, the out-of-the-disk stars could either be generated during the formation process or could initially be in the disk and then perturbed through subsequent dynamical evolution. Self-relaxation of the disk has not had sufficient time to produce the out-of-the-plane population (Alexander et al. 2007; Cuadra et al. 2008), but other mechanisms have been proposed such as scattering by an inward-migrating IMBH (Yu et al. 2007). Currently, our results show that the on-disk and off-disk populations of young stars look very similar outside the central arcsecond $(0.04 \mathrm{pc})$ both in terms of the KLF and the surface density profiles that decreases at larger radii as $\propto r^{-2}$. However, the number of young stars in the disk drops at radii smaller than $0.08 \mathrm{pc}$; and at radii of $\lesssim 0.04 \mathrm{pc}$, none of the observed young S-stars are in the disk (Ghez et al. 2005a; Eisenhauer et al. 2005). This drop in the number of disk stars at small radii may be the result of resonant relaxation or other dynamical processes if the central arcsecond S-stars are a continuation of the disk population (Hopman \& Alexander 2006). Thus, if dynamical evolution produced the off-disk population, then the dynamical process must not be a strong function of radius beyond $0.08 \mathrm{pc}$.

Our distributions show that a potential problem with the single starburst scenario is the presence of the apparent massive star cluster, IRS 13, located $\sim 4^{\prime \prime}$ from the supermassive black hole (Maillard et al. 2004; Schödel et al. 2005). The cluster's orbit is not in the disk plane and, given the proposed mass of IRS 13 ( $>10^{3} M_{\odot}$ ), it is unlikely that it could have been ejected from the disk. However, the definition of IRS 13 as a cluster and the derived mass is based on observations of only 3-4 bright stars and is complicated by enhanced dust and gas emission in the vicinity. More data are needed to determine the total mass of IRS 13 and its relationship to the disk stars.

Our results also have implications for the star-formation mechanism. For both infalling cluster and in situ formation scenarios, we consider whether the observed characteristics of the young stellar disk can be explained. We observe a stellar disk with an out-of-the-disk velocity dispersion of $28 \pm 6 \mathrm{~km} \mathrm{~s}^{-1}$. Additionally, if we consider only orbital solutions within the disk (disk prior), we find that at least 8 of the 22 candidate disk stars have $99.7 \%$ confidence lower limits on the eccentricity of greater than 0.2 . Therefore, any formation scenario should explain not only a single thin stellar disk but also allow for noncircular stellar orbits of some stars in the disk.

First, for the infalling star cluster formation scenario, some of the disk properties we observe are well explained and others appear difficult to reconcile with this model. For instance, eccentric orbits are easily produced. Stars that are stripped from a cluster as it spirals in should have a similar inclination and eccentricity as the cluster itself. Therefore, an infalling cluster with an initially eccentric orbit will produce a disk of stars with similarly eccentric orbits (Berukoff \& Hansen 2006). Previous studies have observed comoving clumps of stars, such as IRS 16SW (Lu et al. 2005) and IRS 13 (Schödel et al. 2005), that appeared to support the infalling cluster formation scenario as they could be the remnant core of the dissipated cluster. We tentatively observe evidence for an overdensity of stars on the front half of the disk at the position of the IRS 16SW comoving 
group. However, this overdensity may be explained by the effects of extinction that reduces the number of young stars identified on the back half of the disk at a given magnitude. The extinction is highly variable throughout the region and the back half of the disk is behind a patch of higher extinction $\left(\Delta A_{K}=0.3-1.4\right.$; Scoville et al. 2003; Schödel et al. 2007). Thus the apparent overdensity on the front half of the disk, corresponding to the IRS $16 \mathrm{SW}$ comoving group, can perhaps be ascribed to differential extinction. More data are needed to confirm the observed disk asymmetry and to determine whether the cause is extinction. Our results yield a steep radial profile for the young stars in the disk, as also found by Paumard et al. (2006), which appears to be inconsistent with the flatter profile expected for an infalling cluster $\left(r^{-0.75}\right.$, Berukoff \& Hansen 2006). We note that mass segregation is observed in massive star clusters that are only a few million years old (Hillenbrand \& Hartmann 1998; Fischer et al. 1998; Stolte et al. 2006). Any mass segregation that existed prior to the cluster's dissolution may impact the observed radial profile as the massive stars would have resided preferentially in the cluster core and would therefore have been deposited at the smallest radii. Thus, the massive stars $\mathrm{O}$ stars that we observe today may have a steeper radial profile than the entire young star population. Additionally, the lack of X-ray emission from premain-sequence stars (Nayakshin \& Sunyaev 2005) is not well explained by an infalling cluster model. A larger and deeper survey for young stars over the central $\sim 5 \mathrm{pc}$ could definitively rule out this scenario if the tidal tails of the disrupted clusters are not detected.

Some theories of in situ star formation take place in a circular gas disk. Such a gas disk can be built up from a steady inward migration of material or from many small cloud-infall events and the disk would circularize prior to becoming massive enough to form stars from self-gravity $\left(>10^{4} M_{\odot}\right)$. Such a formation scenario would most likely produce a steep radial profile in agreement with our observations. Our observations of over $30 \%$ of the candidate disk members with eccentricities greater than 0.2 appears to be inconsistent with an initially circular disk of stars and a normal IMF. A disk of stars on initially circular orbits and with a normal IMF will relax over $6 \mathrm{Myr}$ and produce a thermal distribution of eccentricities with an rms eccentricity of 0.15 or less (Alexander et al. 2007). For such a disk, only 4 out of 22 stars should have eccentricities higher than 0.2, compared with the 8 out of 22 observed when a disk prior is imposed on the primary sample. Therefore, in order for the disk to have been initially circular with a normal IMF, some additional dynamical processing other than self-relaxation is needed. Other possibilities are that the IMF may have been top heavy, the binary fraction may have been extremely high, or IMBHs could have formed, all resulting in faster relaxation to higher eccentricities, but these are not sufficient to explain the out-of-the-disk population of young stars (Alexander et al. 2007; Cuadra et al. 2008). The gas disk formation scenario may be modified (Alexander et al. 2007; Cuadra et al. 2008) to accommodate the observed high stellar eccentricities and out-of-the plane population by building up a massive gas disk in a single cloud infall or a cloud-cloud collision event, in which the clouds are on eccentric orbits (Sanders 1998; Vollmer \& Duschl 2001; Nayakshin et al. 2007). The gas disk would then have a high eccentricity for a short period of time during which stars might form (Alexander et al. 2008). The cloud-cloud collision scenario may yield both a thin stellar disk and a more distributed population of stars at larger radii with a range of angular momenta as a result of the complex interactions and shocks during the collision. It is also conceivable that a cloud-cloud collision scenario might give rise to out-of-the-disk clumps of gas that could form a cluster such as IRS 13. Refined estimates of the eccentricity and inclination distributions of the young stars and more detailed theoretical analysis are needed to investigate the viability of this scenario.

\section{CONCLUSIONS}

In summary, the advent of LGS AO has allowed us to retroactively improve our 11 years astrometric data set used for monitoring stars orbiting our Galactic center. This has increased our proper motion precision, with resulting uncertainties of $\sim 3 \mathrm{~km} \mathrm{~s}^{-1}$, and allowed us, for the first time, to make measurements of and place limits on accelerations for stars outside the central arcsecond out to a radius of 3".5, with typical $3 \sigma$ acceleration limits of -0.19 mas $\mathrm{yr}^{-2}$. By combining our improved stellar positions and proper motions with radial velocity information from the literature, we compute orbits for individual young stars proposed to lie in stellar disks orbiting the supermassive black hole. The orbits for the young stars confirm only a single disk of young stars at a high inclination rotating in a clockwise sense and there is no statistically significant evidence for a second disk. Stars within the well-defined, clockwise disk have an out-of-the-disk velocity dispersion of $28 \pm 6 \mathrm{~km} \mathrm{~s}^{-1}$ and several stars have high eccentricities. These disk properties suggest that star formation may have occurred in a single event, rather than the two events previously needed to explain two stellar disks; however, there are open questions as to how $\sim 50 \%$ of all young stars can be perturbed out of the disk plane and whether the apparent compact cluster, IRS 13, which is not part of the stellar disk, requires a separate star formation or dynamical event. Future directions include (1) obtaining new LGSAO data sets with improved astrometry to measure accelerations for the young stars at all radii and (2) identifying new young stars within the central parsec in order to better constrain the orbital properties of these stars and to study in detail the distribution of eccentricities and semimajor axes for stars both in and out of the disk.

Support for this work was provided by NSF grant AST-0406816, and the NSF Science \& Technology Center for AO, managed by UCSC (AST-9876783). Additional support for J.R.L. was provided by an NSF Graduate Research Fellowship. We would like to thank Brad Hansen and the anonymous referee for helpful comments. The W.M. Keck Observatory is operated as a scientific partnership among the California Institute of Technology, the University of California, and the National Aeronautics and Space Administration. The Observatory was made possible by the generous financial support of the W.M. Keck Foundation.

Facilities: Keck:II (NIRC2), Keck:I (NIRC)

\section{APPENDIX A}

\section{NIRC SPECKLE DISTORTION}

In the speckle data sets, optical distortions, introduced by the NIRC reimager are small near the center of the field of view where Sgr A* was positioned, but grow to dominate the positional uncertainties for stars located more than $\sim 0$ '. 5 from 


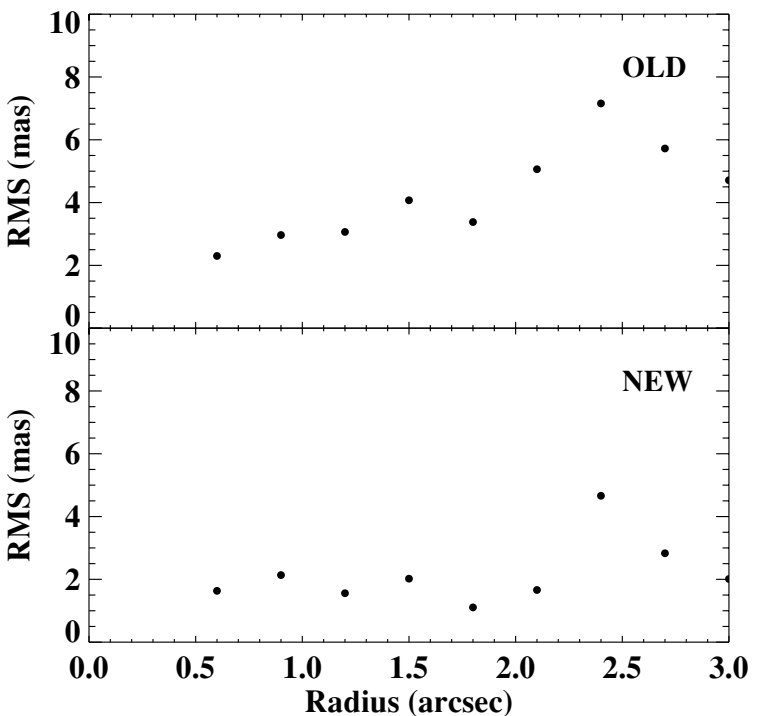

Figure 18. Improvement in positional accuracy at large radii as a result of correcting geometric distortion in speckle data sets. To characterize the systematic positional uncertainty, we take each star at each epoch and calculate the residual positional offset, which is defined as the difference between the measured position and the position as determined by the best-fit velocity $\left(x=x_{o}+v * \Delta t\right)$. Then, the rms of the residuals is calculated across all epochs for each star. All stars' resulting rms values are sorted by the distance between the star and $\mathrm{Sgr} \mathrm{A}^{*}$ (which was at the center of the images) and then averaged over radius bins of 0.3 . The radial trend is shown for data prior to the new distortion correction (top) and after the new distortion correction (bottom).

Sgr A* (see Figure 18 and Section 3.1). Now, utilizing images of the Galactic center obtained with NIRC2, which has optical distortions characterized at the $\sim 2$ mas level (Ghez et al. 2008), we can for the first time, similarly quantify and correct the optical distortions in the NIRC reimager speckle data sets.

Images of the Galactic center were obtained with both NIRC and NIRC2 on consecutive nights during July 2004 and the NIRC2 images were used as a reference coordinate system. The individual NIRC speckle exposure times are only $0.1 \mathrm{~s}$ and have insufficient $\mathrm{S} / \mathrm{N}$ to detect more than the brightest five stars. Exposures were obtained in sets of 100 and each set is combined to produce a single image in which approximately 100 stars are detected. It is assumed that the images are mostly stationary on the NIRC detector during each set of exposures. For each stacked image, the stars' positions are compared to those in the NIRC2 image and the offsets are mapped into NIRC detector coordinates (see Figure 19, left). In this fashion, a distortion map is built up from many stacks of images which are dithered and rotated such that stars fall on many different positions on the detector. The distortion solution was obtained by fitting the distortion map with polynomials of the form

$$
\begin{aligned}
& \left(x^{\prime}+128\right)=a_{0}+a_{1}(x-128)+a_{2}(y-128) \\
& \left(y^{\prime}+128\right)=b_{0}+b_{1}(x-128)+b_{2}(y-128),
\end{aligned}
$$

where the best-fit distortion parameters are listed in Table 5. The new distortion solution improves the rms residual errors per stack by a factor of 3 to $\sim 3$ mas (Figure 20), which is further reduced in the final image by averaging the dithered stacks. Higher-order polynomial terms did not sufficiently improve the fit to warrant inclusion. The above solution is applied after the initial application of the standard NIRC distortion correction. The map of positional differences between stars in the NIRC and NIRC2 images before and after the NIRC reimager distortion correction is shown in Figure 19 (right). The
Table 5

NIRC Reimager Distortion Coefficients

\begin{tabular}{lrc}
\hline \hline $\mathrm{i}$ & \multicolumn{1}{c}{$X\left(a_{i}\right)$} & $Y\left(b_{i}\right)$ \\
\hline 0 & $1.713 \times 10^{-2}$ & $-2.654 \times 10^{-2}$ \\
1 & $9.957 \times 10^{-1}$ & $-1.759 \times 10^{-3}$ \\
2 & $-3.371 \times 10^{-3}$ & 1.004 \\
\hline
\end{tabular}

resulting radial dependence on the rms positional uncertainty is greatly improved and is shown in Figure 18, which plots many stars' rms residual offset from their best-fit proper motions across all epochs. In the final analysis of the speckle data, the relative astrometric uncertainty is $\sim 2$ mas.

\section{APPENDIX B}

\section{ANALYTIC ORBIT EQUATIONS}

The orbit of a star in a known point source potential can be derived from a single measurement of a star's orbital state vector. At epoch $t_{\text {ref }}$, the orbital state vector is usually described by the star's position, $\vec{r}$, and velocity, $\vec{v}$, relative to the central mass. For the analysis in this paper, the state vector is estimated using measurements of the three-dimensional velocity, $\vec{v}=$ $\left[v_{x}, v_{y}, v_{z}\right]$, and the projected position, $\vec{r}_{2 \mathrm{D}}=[x, y]$, and $z$ is derived from the radial acceleration on the plane of the sky. For brevity, we have removed the ref subscript notation and all of the above variables are measured at $t_{\text {ref }}$. Orbital trajectories are then inferred from conservation of energy, specific angular momentum, and eccentricity $(\epsilon, \vec{h}, \vec{e})$, which are related by $\vec{e} \cdot \vec{h}=0$ and $|e|^{2}-1=2 \epsilon h^{2} / G M$ giving five constants of motion plus an undetermined reference time. Equivalently, the orbital trajectory can be expressed using the standard Keplerian orbital elements: period $(P)$, eccentricity $(e)$, time of periapse passage $\left(T_{\circ}\right)$, inclination $(i)$, position angle of the ascending node $(\Omega)$, and the longitude of periapse ( $\omega$; see Equations (B5), (B6), (B7), (B8), (B9), (B17) and Ghez et al. 2005a, for detailed descriptions of these orbital parameters). The three-dimensional position and velocity state vectors can be used to calculate the orbit of the star around the black hole (by algebraic manipulation of Kepler's laws). Here, we present the analytic expressions used to compute the orbital elements from the state vectors.

Orbit determination for the young stars in our sample is tractable because the mass and position of the black hole are determined by independent means, namely the well-determined orbits of stars much closer to the black hole. The coordinate system is set such that $\operatorname{Sgr} A^{*}$ resides at the origin, $\hat{x}$ and $\hat{y}$ increase with right ascension and declination, and $\hat{z}$ increases with the line-of-sight distance from the Earth to Sgr A* with $z=0$ at the location of the black hole. Combining the two state vectors, $\vec{r}$ and $\vec{v}$, and the black hole mass, there are three intermediate vectors that describe the geometry of the orbit both in three dimensions and projected onto the plane of the sky. These are (1) the specific angular momentum vector, $\vec{h}$, which points normal to the plane of the orbit, (2) the eccentricity vector, $\vec{e}$, which points in the direction of periapse, and (3) the ascending node vector, $\vec{\Omega}$, which points to where the star passes through the plane of the sky moving away from us, and are given by

$$
\begin{aligned}
\vec{h} & =\vec{r} \times \vec{v} \\
\vec{e} & =\frac{\vec{v} \times \vec{h}}{G M}-\frac{\vec{r}}{|\vec{r}|} \\
\vec{\Omega} & =\vec{h} \times \hat{z} .
\end{aligned}
$$



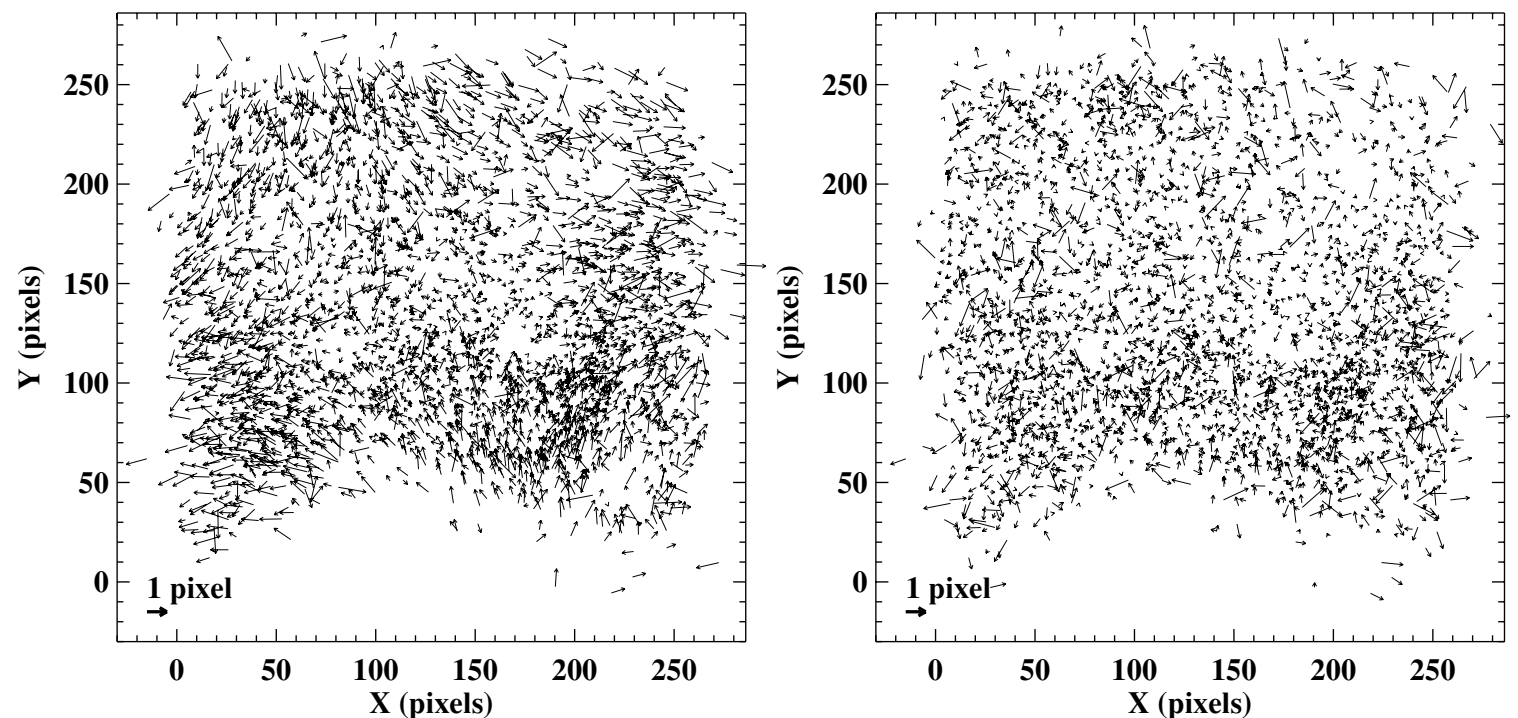

Figure 19. Map of the positional differences between stars observed near-simultaneously with NIRC and NIRC2. The maps are plotted in the original NIRC detector coordinates and show residuals before (left; (a)) and after (right; (b)) the NIRC reimager distortion solution.

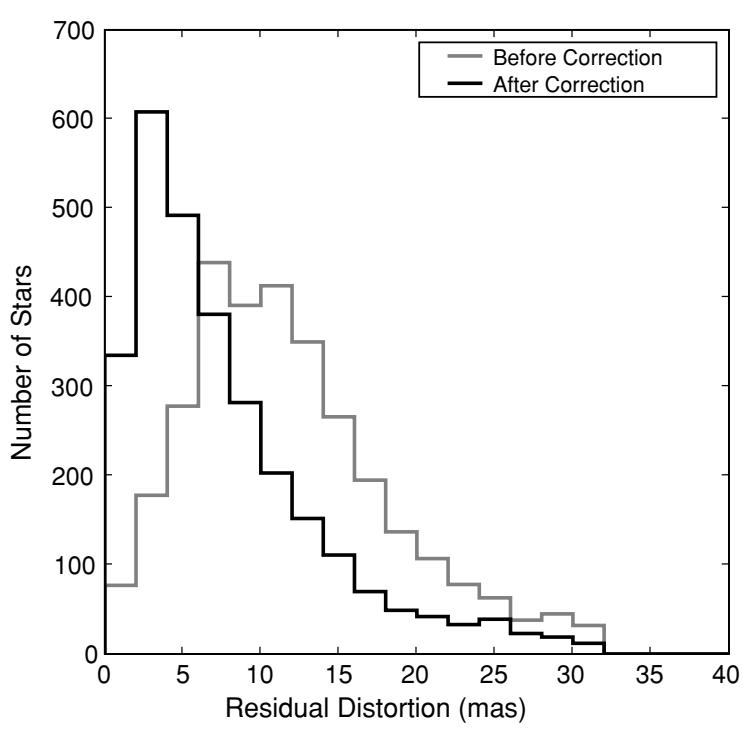

Figure 20. Distribution of the residuals before (gray) and after (black) correcting for the NIRC image converter distortion. Residuals are calculated by comparing a star's position in each NIRC image stack to the position in the LGS AO/ NIRC2 image. These residuals are further reduced in the final image because the stacks are dithered small amounts on the detector and residual distortion can be averaged out if it is randomly oriented over the scale of the dither.

The semimajor axis can also be calculated as an intermediate quantity

$$
a=\left(\frac{2}{|\vec{r}|}-\frac{|\vec{v}|^{2}}{G M}\right)^{-1}
$$

Then, the five standard orbital parameters that describe the shape and period of the orbit are then

$i=\arccos \left(\frac{-\vec{h} \cdot \hat{z}}{|\vec{h}|}\right)$

$e=|\vec{e}|$

$\omega=\arccos \left(\frac{(\hat{z} \times \vec{h}) \cdot \vec{e}}{|\hat{z} \times \vec{h}||\vec{e}|}\right) \quad($ if $\vec{e} \cdot \hat{z}<0$ then $\omega=2 \pi-\omega)$

$$
\begin{aligned}
& \Omega=\arctan \left(\frac{\vec{\Omega} \cdot \hat{x}}{\vec{\Omega} \cdot \hat{y}}\right) \\
& \left(\frac{P}{[y r]}\right)=\sqrt{\left(\frac{a}{[A U]}\right)^{3}\left(\frac{\left[M_{\odot}\right]}{M}\right)},
\end{aligned}
$$

where $i=0$ if the orbit is in the plane of the sky and $\Omega$ is measured east $(\hat{x})$ of north $(\hat{y})$. The remaining orbital parameter is the epoch of periapse passage and can be computed in a number of different ways. We first compute several intermediate quantities of interest such as the Thiele-Innes constants (A, B, $\mathrm{C}, \mathrm{F}, \mathrm{G}, \mathrm{H})$, and the eccentric anomaly as shown below:

$$
\begin{aligned}
A & =a(\cos \omega \cos \Omega-\sin \omega \sin \Omega \cos i) \\
B & =a(\cos \omega \sin \Omega+\sin \omega \cos \Omega \cos i) \\
F & =a(-\sin \omega \cos \Omega-\cos \omega \sin \Omega \cos i) \\
G & =a(-\sin \omega \sin \Omega+\cos \omega \cos \Omega \cos i) \\
\cos E & =\frac{G r_{y}-F r_{x}}{A G-B F}+e \\
\sin E & =\frac{A r_{x}-B r_{y}}{A G-B F} \frac{1}{\sqrt{1-e^{2}}} \\
E & =\arctan \left(\frac{\sin E}{\cos E}\right) .
\end{aligned}
$$

And finally the epoch of periapse passage are calculated from these intermediate quantities using

$$
T_{o}=t_{\text {ref }}-\frac{P}{2 \pi}(E-e \sin E) .
$$

\section{APPENDIX C}

\section{$K$ METRIC}

The previously proposed planes were derived by minimizing a metric that Levin $\&$ Beloborodov (2003) call $\chi^{2}$, but we call $K$, and which is defined as

$$
K=\frac{1}{N-1} \sum_{i=1}^{N} \frac{\left(\vec{n} \cdot \vec{v}_{i}\right)^{2}}{\left(n_{x} \sigma_{v_{x, i}}\right)^{2}+\left(n_{y} \sigma_{v_{y, i}}\right)^{2}+\left(n_{z} \sigma_{v_{z, i}}\right)^{2}},
$$


where $N$ is the number of stars, $\vec{v}_{i}$ is the velocity of each star, $\sigma_{v_{x, i}}$, $\sigma_{v_{y, i}}, \sigma_{v_{z, i}}$ are the velocity uncertainties for each star, and $\vec{n}$ is the normal vector to the disk plane that is found in the fitting process. This metric is used to find, statistically, the best-fit common orbital plane from the velocity vectors of a sample of stars. The $K$ metric suffers from several shortcomings. First, the $K$ metric is described as a $\chi^{2}$ metric; however, standard $\chi^{2}$ minimization takes the form of $(\text { data }- \text { model })^{2} /(\text { data errors })^{2}$ where the data errors have no dependency on the model parameters. The $K$ metric includes the model parameters in the data-error term and does not necessarily have an expectation value of 1 for normal errors. The appropriate function to minimize in order to find the best-fit common orbital plane can be derived from maximum likelihood theory if we assume that the likelihood function is given by

$$
L=\prod_{i=1}^{N} \frac{1}{\sqrt{2 \pi \sigma_{i}^{2}}} \exp \left[-\frac{\left(\vec{n} \cdot \vec{v}_{i}\right)^{2}}{2 \sigma_{i}^{2}}\right]
$$

where $\sigma_{i}$ depends on the disk model parameters that are being sought by

$$
\sigma_{i}^{2}=\left(n_{x} \sigma_{v_{x, i}}\right)^{2}+\left(n_{y} \sigma_{v_{y, i}}\right)^{2}+\left(n_{z} \sigma_{v_{z, i}}\right)^{2} .
$$

Standard practice is then to take the logarithm of the likelihood, $L$, and minimize the resulting function in Equation (C5) in order to find the best-fit disk model parameters. The above likelihood function then becomes

$$
\begin{aligned}
\ln L & =-\frac{N}{2} \ln (2 \pi)-\sum_{i=1}^{N} \ln \sigma_{i}+\sum_{i=1}^{N}-\frac{\left(\vec{n} \cdot \vec{v}_{i}\right)^{2}}{2 \sigma_{i}^{2}} \\
-2 \ln L & =N \ln (2 \pi)+2 \sum_{i=1}^{N} \ln \sigma_{i}+\sum_{i=1}^{N} \frac{\left(\vec{n} \cdot \vec{v}_{i}\right)^{2}}{\sigma_{i}^{2}}
\end{aligned}
$$

and the first two terms are constant and do not factor into finding an extremum in the above equation. The third term on the righthand side is the $K$ metric previously used to determining the disk parameters. However, the second term on the right-hand side also depends on the free parameters in $\vec{n}$ and must be included in the minimization process. This extra term that has not previously been included in the disk fitting process has the full form

$$
\ln \sqrt{\left(n_{x} \sigma_{v_{x, i}}\right)^{2}+\left(n_{y} \sigma_{v_{y, i}}\right)^{2}+\left(n_{z} \sigma_{v_{z, i}}\right)^{2}}
$$

and standard chi-squared probability functions cannot be applied. Second, even when accounting for the extra term, the metric can still introduce substantial bias. In particular, radial velocity uncertainties, $\sigma_{v_{z i}}$, are larger than the proper motion errors by a factor of 2 on average in previous publications. During $K$-minimization, this overweights solutions with a larger $n_{z}$ resulting in a bias against edge-on planes. Finally, in order to properly evaluate the probability of obtaining a given value of the $K$-metric by random chance, one must perform simulations of an isotropic distribution of stars. However, such simulations are extremely sensitive to the input distribution of semimajor axes and eccentricities which are not yet well constrained by observations. Thus, when utilizing such statistical tests for finding a common orbital plane, it is difficult to compare to the null hypothesis - an isotropic distribution of stars - and to quantify the significance of a disk.

\section{REFERENCES}

Alexander, T. 2005, Phys. Rep., 419, 65

Alexander, R. D., Armitage, P. J., Cuadra, J., \& Begelman, M. C. 2008, ApJ, 674,927

Alexander, R. D., Begelman, M. C., \& Armitage, P. J. 2007, ApJ, 654, 907

Alexander, T., \& Morris, M. 2003, ApJ, 590, L25

Allen, D. A., Hyland, A. R., \& Hillier, D. J. 1990, MNRAS, 244, 706

Babu, G. J., \& Feigelson, E. D. 1996, in Astrostatistics, ed. G. J. Babu \& E. D. Feigelson (London: Chapman and Hall)

Beloborodov, A. M., Levin, Y., Eisenhauer, F., Genzel, R., Paumard, T., Gillessen, S., \& Ott, T. 2006, ApJ, 648, 405

Bender, R., et al. 2005, ApJ, 631, 280

Berukoff, S. J., \& Hansen, B. M. S. 2006, ApJ, 650, 901

Blum, R. D., Depoy, D. L., \& Sellgren, K. 1995, ApJ, 441, 603

Cuadra, J., Armitage, P. J., \& Alexander, R. D. 2008, MNRAS, 388, L64

Davies, M. B., Blackwell, R., Bailey, V. C., \& Sigurdsson, S. 1998, MNRAS, 301,745

Davies, M. B., \& King, A. 2005, ApJ, 624, L25

Diolaiti, E., Bendinelli, O., Bonaccini, D., Close, L., Currie, D., \& Parmeggiani, G. 2000, A\&AS, 147, 335

Dressler, A. 1980, ApJ, 236, 351

Eckart, A., \& Genzel, R. 1996, Nature, 383, 415

Eckart, A., Genzel, R., Ott, T., \& Schödel, R. 2002, MNRAS, 331, 917

Eisenhauer, F., et al. 2005, ApJ, 628, 246

Fischer, P., Pryor, C., Murray, S., Mateo, M., \& Richtler, T. 1998, AJ, 115, 592

Fruchter, A. S., \& Hook, R. N. 2002, PASP, 114, 144

Genzel, R., Pichon, C., Eckart, A., Gerhard, O. E., \& Ott, T. 2000, MNRAS, 317,348

Genzel, R., Thatte, N., Krabbe, A., Kroker, H., \& Tacconi-Garman, L. E. 1996, ApJ, 472, 153

Genzel, R., et al. 2003, ApJ, 594, 812

Gerhard, O. 2001, ApJ, 546, L39

Ghez, A. M., Klein, B. L., Morris, M., \& Becklin, E. E. 1998, ApJ, 509, 678

Ghez, A. M., Morris, M., Becklin, E. E., Tanner, A., \& Kremenek, T. 2000, Nature, 407, 349

Ghez, A. M., Salim, S., Hornstein, S. D., Tanner, A., Lu, J. R., Morris, M., Becklin, E. E., \& Duchêne, G. 2005a, ApJ, 620, 744

Ghez, A. M., et al. 2003, ApJ, 586, L127

Ghez, A. M., et al. 2005b, ApJ, 635, 1087

Ghez, A. M., et al. 2008, ApJ,, 689, 1044

Goodman, J. 2003, MNRAS, 339, 937

Górski, K. M., Hivon, E., Banday, A. J., Wandelt, B. D., Hansen, F. K., Reinecke, M., \& Bartelmann, M. 2005, ApJ, 622, 759

Gürkan, M. A., \& Rasio, F. A. 2005, ApJ, 628, 236

Hansen, B. M. S., \& Milosavljević, M. 2003, ApJ, 593, L77

Hillenbrand, L. A., \& Hartmann, L. W. 1998, ApJ, 492, 540

Hopman, C., \& Alexander, T. 2006, ApJ, 645, 1152

Hornstein, S. D. 2007, PhD thesis, UCLA

Kim, S. S., Figer, D. F., \& Morris, M. 2004, ApJ, 607, L123

Kim, S. S., \& Morris, M. 2003, ApJ, 597, 312

Kolykhalov, P. I., \& Syunyaev, R. A. 1980, Sov. Astron. Lett., 6, 357

Krabbe, A., Genzel, R., Drapatz, S., \& Rotaciuc, V. 1991, ApJ, 382, L19

Krabbe, A., et al. 1995, ApJ, 447, L95

Lee, H. M. 1996, in IAU Symposium 169, Unsolved Problems of the Milky Way, ed. L. Blitz \& P. J. Teuben (Dordrecht: Kluwer), 215

Levin, Y. 2007, MNRAS, 374, 515

Levin, Y., \& Beloborodov, A. M. 2003, ApJ, 590, L33

Lin, D. N. C., \& Pringle, J. E. 1987, MNRAS, 225, 607

Lu, J. R., Ghez, A. M., Hornstein, S. D., Morris, M., \& Becklin, E. E. 2005, ApJ, 625, L51

Maillard, J. P., Paumard, T., Stolovy, S. R., \& Rigaut, F. 2004, A\&A, 423, 155

Matthews, K., Ghez, A. M., Weinberger, A. J., \& Neugebauer, G. 1996, PASP, 108,615

Matthews, K., \& Soifer, B. T. 1994, Exp. Astron., 3, 77

McMillan, S. L. W., \& Portegies Zwart, S. F. 2003, ApJ, 596, 314

Milosavljević, M., \& Loeb, A. 2004, ApJ, 604, L45

Morris, M. 1993, ApJ, 408, 496

Morris, M., \& Serabyn, E. 1996, ARA\&A, 34, 645

Najarro, F., Krabbe, A., Genzel, R., Lutz, D., Kudritzki, R. P., \& Hillier, D. J. 1997, A\&A, 325, 700

Nayakshin, S., \& Cuadra, J. 2005, A\&A, 437, 437

Nayakshin, S., Cuadra, J., \& Springel, V. 2007, MNRAS, 379, 21

Nayakshin, S., Dehnen, W., Cuadra, J., \& Genzel, R. 2006, MNRAS, 366,1410

Nayakshin, S., \& Sunyaev, R. 2005, MNRAS, 364, L23

Ott, T. 2003, PhD thesis, Max-Planck Institut für Extraterrestrische Physik 
Paumard, T., et al. 2006, ApJ, 643, 1011

Portegies Zwart, S. F., McMillan, S. L. W., \& Gerhard, O. 2003, ApJ, 593,352

Rafelski, M., Ghez, A. M., Hornstein, S. D., Lu, J. R., \& Morris, M. 2007, ApJ, 659,1241

Sanders, R. H. 1992, Nature, 359, 131

Sanders, R. H. 1998, MNRAS, 294, 35

Schödel, R., Eckart, A., Iserlohe, C., Genzel, R., \& Ott, T. 2005, ApJ, 625, L111

Schödel, R., Ott, T., Genzel, R., Eckart, A., Mouawad, N., \& Alexander, T. 2003, ApJ, 596, 1015

Schödel, R., et al. 2007, A\&A, 469, 125
Schödel, R., et al. 2002, Nature, 419, 694

Scoville, N. Z., Stolovy, S. R., Rieke, M., Christopher, M., \& Yusef-Zadeh, F. 2003, ApJ, 594, 294

Shlosman, I., \& Begelman, M. C. 1989, ApJ, 341, 685

Stolte, A., Brandner, W., Brandl, B., \& Zinnecker, H. 2006, AJ, 132, 253

Tamblyn, P., Rieke, G. H., Hanson, M. M., Close, L. M., McCarthy, D. W., \& Rieke, M. J. 1996, ApJ, 456, 206

van Dam, M. A., et al. 2006, PASP, 118, 310

Vollmer, B., \& Duschl, W. J. 2001, A\&A, 377, 1016

Wizinowich, P. L., et al. 2006, PASP, 118, 297

Yu, Q., Lu, Y., \& Lin, D. N. C. 2007, ApJ, 666, 919 